Mishor et al

\title{
Sniffing the Human Body-Volatile Hexadecanal Blocks Aggression in Men but Triggers Aggression in Women
}

Eva Mishor ${ }^{1,2^{*}}$, Daniel Amir ${ }^{1,2}$, Tali Weiss ${ }^{1,2}$, Danielle Honigstein ${ }^{1,2}$, Aharon Weissbrod ${ }^{1,2}$, Ethan Livne ${ }^{1,2}$, Lior Gorodisky ${ }^{1,2}$, Shiri Karagach ${ }^{1,2}$, Aharon Ravia ${ }^{1,2}$, Kobi Snitz ${ }^{1,2}$, Diyala Karawani $^{1,2}$, Rotem Zirler ${ }^{1,2}$, Reut Weissgross ${ }^{1,2}$, Timna Soroka ${ }^{1,2}$, Yaara Endevelt-Shapira ${ }^{1,2}$, Shani Agron ${ }^{1,2}$, Liron Rozenkrantz ${ }^{1,2}$, Netta Reshef ${ }^{1,2}$, Edna Furman-Haran $^{1}$, Heinz Breer ${ }^{3}$, Joerg Strotmann ${ }^{3}$, Noam Sobel ${ }^{1,2^{*}}$

${ }^{1}$ Azrieli National Center for Brain Imaging and Research and ${ }^{2}$ Department of Neurobiology, Weizmann Institute of Science, Rehovot, Israel. ${ }^{3}$ Institute of Physiology, University of Hohenheim, Stuttgart, Germany

*Correspondence at:

eva.mishor@weizmann.ac.il

noam.sobel@weizmann.ac.il 
Mishor et al

\begin{abstract}
Body-volatiles can effectively trigger or block conspecific aggression in terrestrial mammals. Here we tested whether hexadecanal (HEX), a human body-volatile implicated as a mammalian-wide social cue, impacts human aggression. Using validated behavioural paradigms, we observed a remarkable dissociation: sniffing HEX blocked aggression in men, but triggered aggression in women. Next, using functional brain imaging, we uncovered a pattern of brain activity mirroring behaviour: In both men and women, HEX increased activity in the left angular gyrus, an area implicated in perception of social cues. Hex then modulated functional connectivity between the angular gyrus and a brain network implicated in social appraisal (temporal pole) and aggressive execution (amygdala and orbitofrontal cortex) in a sex-dependent manner consistent with behaviour: increasing connectivity in men, but decreasing connectivity in women. These findings implicate sex-specific social chemosignaling at the mechanistic heart of human aggressive behaviour.
\end{abstract}


Mishor et al

\section{Introduction}

Terrestrial mammalian aggressive behavior can be triggered or blocked by social odors (1-4). For example, a rabbit mother will attack and even kill her pups if they are tainted with the body-odor of a stranger female (5), and two specific volatile components in mouse urine trigger fighting between males (6). Such aggression-triggering chemosignals are not all volatile: specific major urinary proteins (MUPs) can also trigger aggression, an effect mediated by the accessory olfactory system (7). In turn, chemosignals not only trigger aggression, they can also block it. For example, tainting a pig with the known volatile reproductive pheromone androstenone (5a-Androst-16-en-3-one) blocks conspecific aggression towards the tainted pig (8). Also, a mouse tear-bound peptide blocks male mouse aggression towards mouse pups tainted with the peptide (9). Such tear-bound aggressionblocking chemosignals may be a mammalian-wide phenomenon: male mole-rats cover themselves with their own harderian secretions, and this blocks aggression towards them from dominant male conspecifics (10). Relatedly, sniffing human tears reduces testosterone in men $(11,12)$, but the impact of this on aggressive behavior has yet to be investigated. To conclude, both volatile and non-volatile chemosignals can either trigger or block mammalian aggression.

Like all mammals, humans engage in reactive aggression from a very early age (13), and throughout life (14). Although aggression is a major factor in the human condition, the mechanistic neural substrates of human aggression are not well-understood (15-17). Could human aggression mechanisms be tied to chemosignaling mechanisms as they are in all other terrestrial mammals? Two studies have suggested that humans emit aggression-specific body-odors $(18,19)$, but whether and how human aggressive behaviour is then impacted by social chemosignals remains unknown. To test the hypothesis that a social chemosignal can modulate human aggression through modulation of neural activity we used standard behavioural and neuroimaging aggression paradigms with and without a concurrent social chemosignal. As a social chemosignal, we tested Hexadecanal (HEX), a volatile long-chain aliphatic aldehyde, first identified as a social-buffering agent in mice (20). Although speciesspecificity has been considered a hallmark of social chemosignaling (21), some signaling molecules may be conserved across species, with either similar or different signal meaning $(22,23)$. Because the mouse receptor for HEX (OR37B) is highly conserved across mammals, 
Mishor et al

it has been suggested that HEX may be one such cross-species conserved signaling molecule (24). Humans indeed express an OR37 receptor orthologue $(25,26)$, and indeed emit HEX in feces, skin, and breath $(27,28)$. Moreover, exposure to HEX reduced startle-responses in humans (29), implicating impact on arousal. With all this in mind, we tested whether and how HEX impacts human aggression.

\section{Results}

\section{HEX blocked aggression in men but triggered aggression in women}

To gauge aggression, we used a modified Taylor aggression paradigm (TAP), a well-established measure of human aggressive behaviour (30-33). A total of 127 participants (67 men, mean age $25.48 \pm 3.46$, range $21-34$ ) played the game in a double-blind between-subjects design, half concurrent with exposure to HEX obscured in carrier (Eugenol), and half concurrent with exposure to carrier alone (Figure 1A). To ask whether the addition of HEX was associated with an explicit percept, participants used a visual-analogue scale (VAS) to rate the stimuli along the primary dimensions of human olfactory perception; namely intensity and pleasantness (34) (Figure 1B, 1C). Because odorant ratings did not distribute normally (Pleasantness: Shapiro-Wilk $W=0.98, p=0.001$, Intensity: $W=0.96, p=0.002$ ) we applied a linear mixed model with factors of Sex and Odor and random effects of participant, which revealed that HEX had no impact on perceived odorant pleasantness (Sex: $F(1,123)=1.13, p=0.29$, Odor: $F(1,123)=0.12, p=0.73$, Sex $x$ Odor: $F(1,123)=0.10, p=0.7)$, and no overall impact on perceived intensity, but a marginal yet statistically significant interaction (Sex: $F(1,123)=$ 3.66, $p=0.06$, Odor: $F(1,123)=0.6, p=0.44$, Sex $x$ Odor: $F(1,123)=6.07, p=0.02)$. Post-hoc comparisons (Tukey adjustment for multiple comparisons), however, revealed that this interaction reflected opposing insignificant effects in men and women (Men Control: mean VAS $=-0.09 \pm 0.07$, men HEX: mean VAS $=-0.22 \pm 0.77, t(123)=1.10, p=0.69$. Women Control: mean VAS $=-0.09 \pm 0.60$, women HEX: mean VAS $=0.38 \pm 0.96, t(123)=2.35, p=0.09$ ) (Figure 1D, 1E). In sum, the addition of HEX to the carrier had no impact on perceived stimulus pleasantness, and minimal impact on perceived stimulus intensity. 
bioRxiv preprint doi: https://doi.org/10.1101/2020.09.29.318287; this version posted December 14,2020 . The copyright holder for this preprint (which was not certified by peer review) is the author/funder, who has granted bioRxiv a license to display the preprint in perpetuity. It is made available under aCC-BY-NC-ND 4.0 International license.

Mishor et al

A

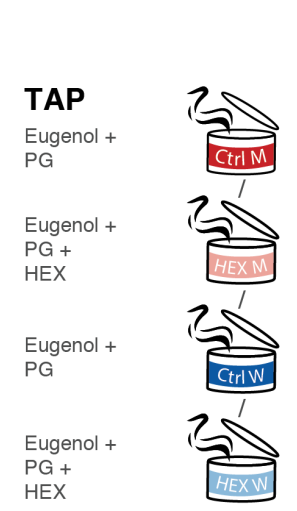

B

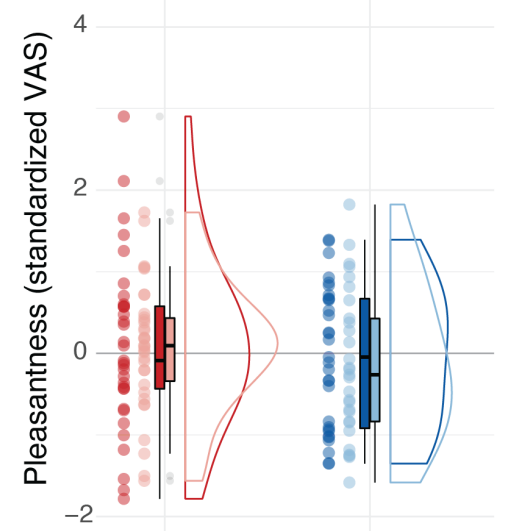

C

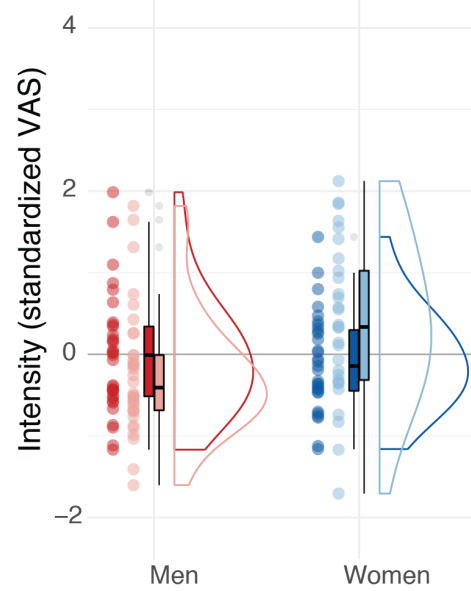

D

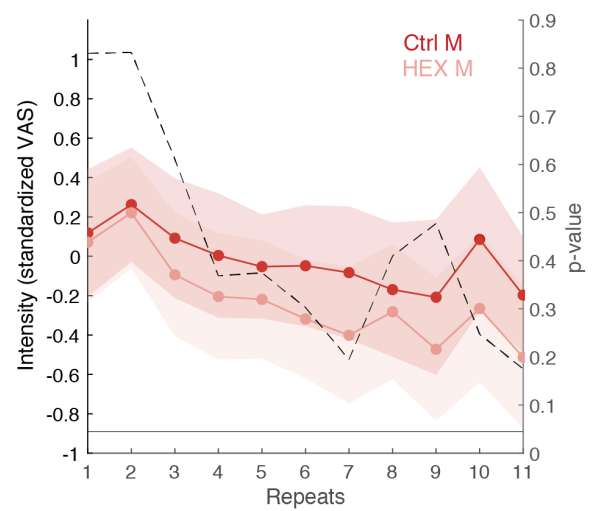

$\mathbf{F}$

\section{FC-PSAP}

$\begin{array}{ccc}\text { Clean } & \text { Mineral } & \text { Mineral } \\ \text { air } & \text { oil } & \text { oil }+ \\ & & \text { HEX }\end{array}$
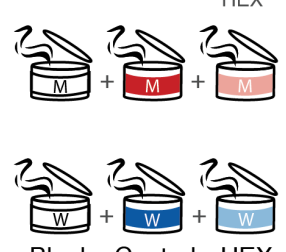

Blank Control HEX

Counterbalanced
G

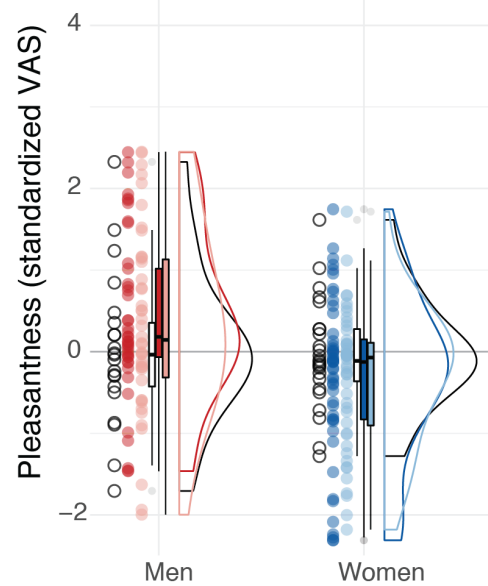

E

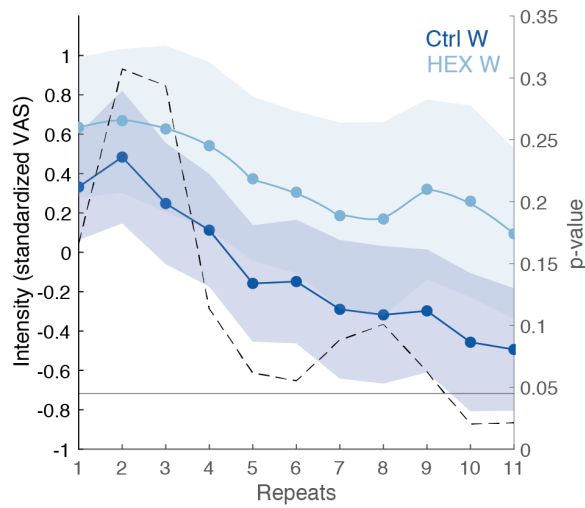

H

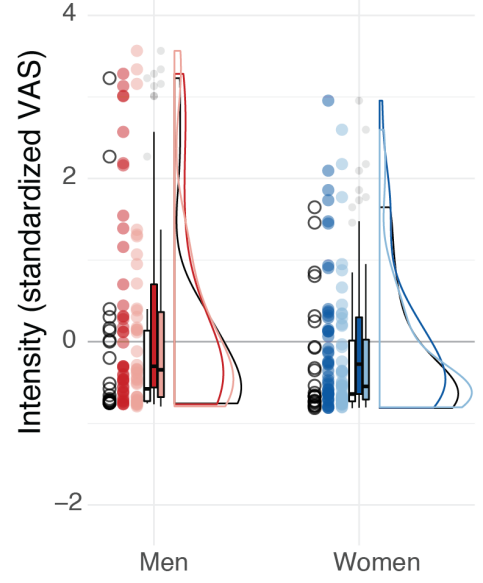

I

J
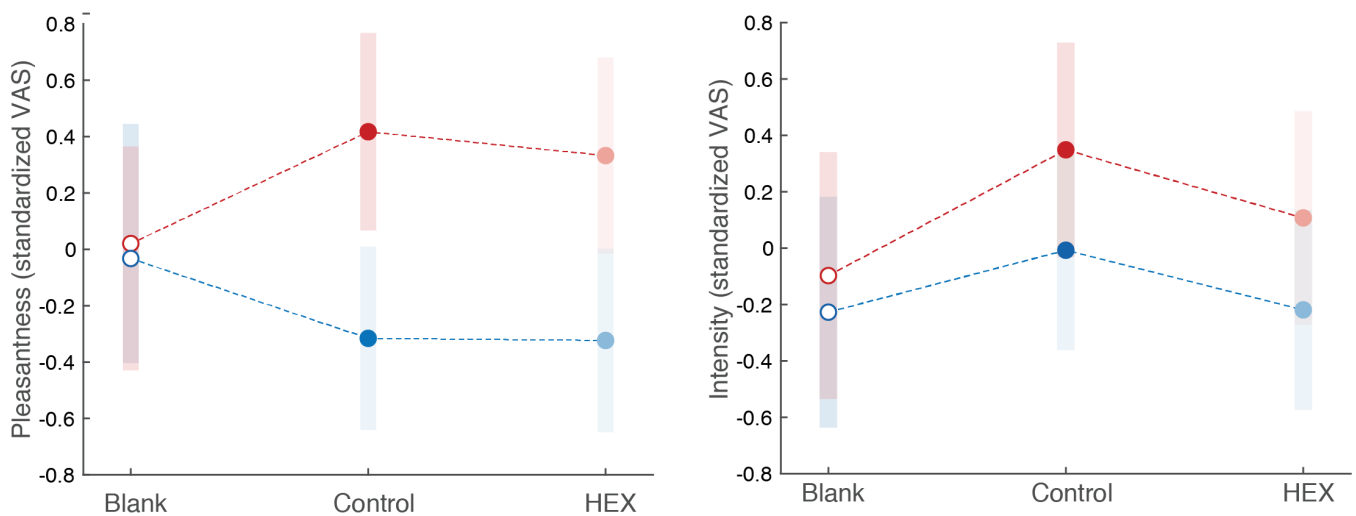
Mishor et al

Figure 1. HEX did not significantly shift stimulus perception

(A) The between-subjects TAP included four groups exposed to either control (100 ul, 10\% eugenol in propylene glycol (PG)) or HEX (100 ul, 0.083M HEX in 10\% eugenol in PG). Control $\mathrm{n}=34 \mathrm{M}$ and $31 \mathrm{~F}, \mathrm{HEX} \mathrm{n}=33 \mathrm{M}$ and 29F. (B) TAP odorant pleasantness ratings along the VAS. Each dot is a participant, the thick horizontal line is the median, the rectangle reflects the interquartile range (25th to the 75th percentiles) and the whiskers are no more than $1.5 *$ IQR of the upper and lower hinges. Outlying points are plotted individually. (C) TAP odorant Intensity ratings along the VAS. Elements as in B. (D) Mean Intensity and confidence interval for 11 consecutive exposures to HEX and Control, in men. The dotted line is the point-by-point p-value for the HEX-Control t-test. The horizontal line represents the significance threshold p-value, corrected for multiple comparisons (Bonferroni correction), set to 0.0045. (E) Same as in $D$, in women. (F) The within-subjects FC-PCAP included two groups: men $(n=25)$ and women $(n=24)$. Both were exposed to Blank (clean air) Control (mineral oil) and HEX (0.083M HEX dissolved in mineral oil). (G) FC-PCAP odorant pleasantness ratings along the VAS. Elements as in B. (H) FC-PCAP odorant intensity ratings along the VAS. Elements as in B. (I) FCPCAP odorant pleasantness ratings mean and confidence interval. (J) FC-PCAP odorant intensity ratings mean and confidence interval.

The TAP begins with a provocation phase where the participant plays an on-screen version of the ultimatum game (35) against a purported game-partner who is in fact a game algorithm (Figure 2A). In each round of the ultimatum game, both "players" are allotted a sum of money ( $\$ 9)$ that they can keep if they agree on how to distribute it between them. The game is programmed such that the purported partner agrees only to distributions that significantly discriminate against the participant. Five such rounds served as an effective provocation. At this point, there were no differences between the groups (Supplementary Figure 1; Supplementary Figure 2). Following this, is an aggression discharge phase where the participant is again misled to believe they are playing against the same partner (who previously provoked them), but now in a reaction-time task where they compete to identify a change in shape of a target. The first to react is then allowed to blast his/her opponent with a loud noise blast. The volume applied by the participant is taken as a measure of aggression $(36,37)$ (Figure 2B). The game was rigged such that the participant was faster than the fictitious opponent on 16 of 27 rounds, and in rounds where the fictitious opponent was faster, the participant endured noise-blasts randomly ranging in volume. 
A

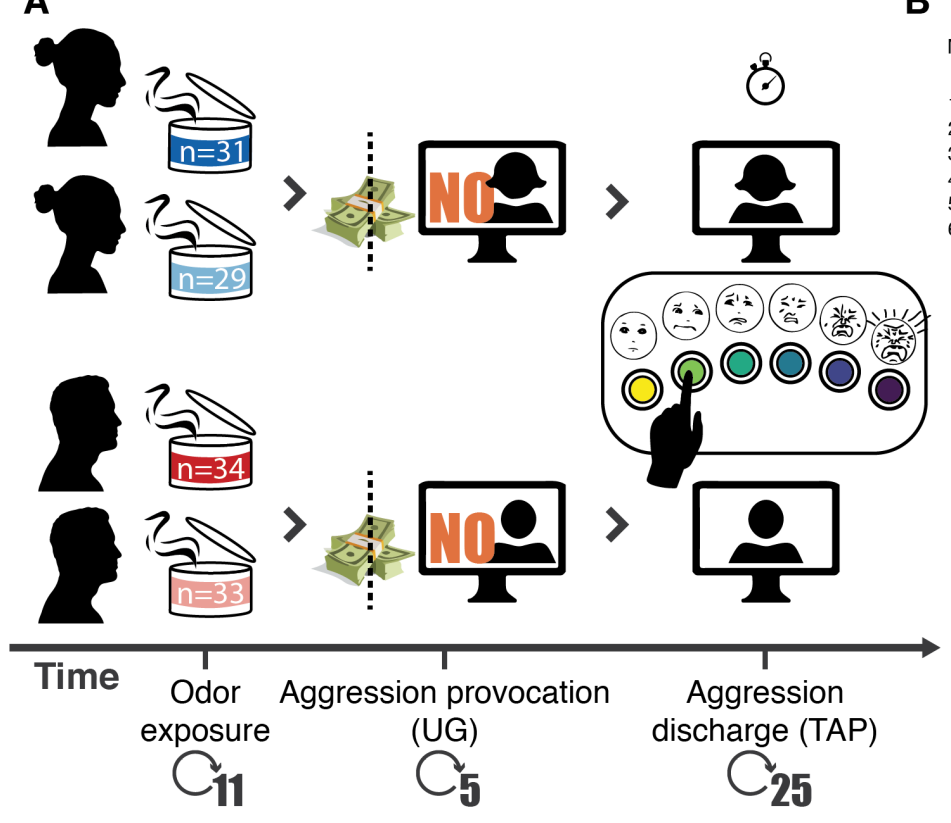

B

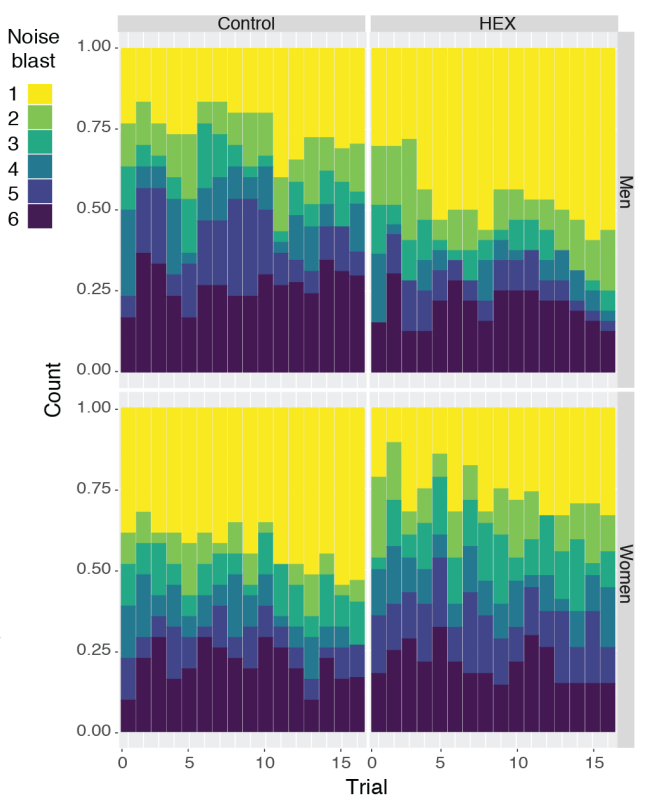

Figure 2. Path to provoking and gauging human aggressive behaviour

(A) In a between-subjects design, participants were exposed to an odorant (HEX or control), then played a game where their online partner was unfair towards them in monetary distribution (provocation), and then another game where they could blast that same (nonexistent) person with noise-blasts (aggression discharge). (B) The complete distribution of noise-blasts applied in the study by men $(n=67)$ and women $(n=60)$ under HEX or control (yellow $=$ mild, purple $=$ harsh).

Noise-blast volume was entered into a repeated-measures ordinal logistic regression analysis with factors of Odorant (HEX/Control) and Sex (men/women), and random effect of Participant. We observed a trend towards an effect of Odorant $(Z=1.84, p=0.07)$, no effect of $\operatorname{Sex}(Z=1.63, p=0.10)$, and a significant Odorant $X$ Sex interaction $(Z=2.03, p=0.04$, effect size: generalized $\left.\eta^{2}=0.04\right)$. This significant interaction reflected that $\mathrm{HEX}$ significantly lowered aggression in men (men Hex: $n=34$, mean blast vol $=2.76 \pm 2.03$, men Control: $n=33$, mean blast vol $=3.60 \pm 1.98$, Mann-Whitney (trials compacted as repeated measure), $Z=5.855, p$ $<0.0001$, effect size $r=0.7$ ), yet significantly increased aggression in women (women Hex: $n$ $=29$, mean blast vol $=3.40 \pm 1.91$, women Control: $\mathrm{n}=31$, mean blast vol $=2.96 \pm 2.03$, MannWhitney (trials compacted as repeated measure), $Z=3.655, p=0.0003$, effect size $r=0.47$ ) (Figure 3A).

To gain further insight into the dynamics of aggressive behaviour in the TAP, we looked at the 16 repeated trials separately. When depicting the trial-by-trial mean, there is an apparent 
Mishor et al

small yet highly consistent difference between the conditions in both sexes (Figure 3B, 3C). To again quantify this, we compared the trial-by-trial means using a Mann-Whitney test, which provided a trial-wise $\mathrm{Z}$ value. An independent t-test on the trial-by-trial z-values again revealed a significant effect of condition within sex: significantly decreasing aggression in men exposed to HEX (Trial-wise blast volume: control $=3.57 \pm 0.34, \mathrm{HEX}=2.91 \pm 0.39, \mathrm{t}(16)=-$ $6.37, p<10^{-5}$ ) yet significantly increasing it in women (Trial-wise blast volume: control $=2.84$ $\pm 0.55, \mathrm{HEX}=3.34 \pm 0.33, \mathrm{t}(15)=4.70, \mathrm{p}<0.0003$ ) (these numbers differ from the previous analysis because they reflect trial-wise blast volume, whereas the previous analysis reflected participant-wise blast volume), again indicating a significant dissociation between the two sexes $\left(t(15)=-11.18, p<10^{-7}\right)$ (these differences again re-emerged in a validation permutation test (Supplementary Figure 3)). Behavioural differences were not associated with perceptual valence differences between the two conditions (Figure 1), were not related to vigilance (Supplementary Figure 4), and were not reflected in group-levels of Cortisol or Testosterone (Supplementary Figure 5). In sum, three separate analyses of the TAP implied that exposure to HEX reduced aggression in men but increased aggression in women. 
A

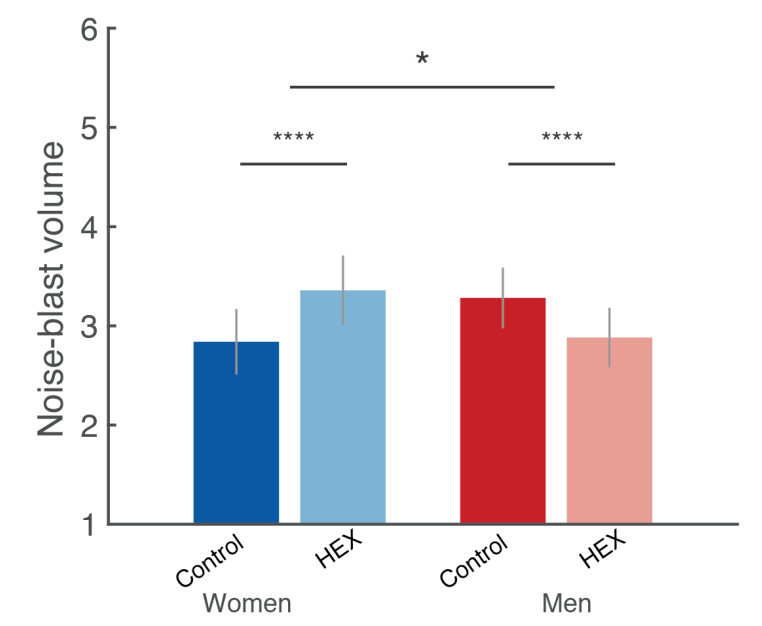

C

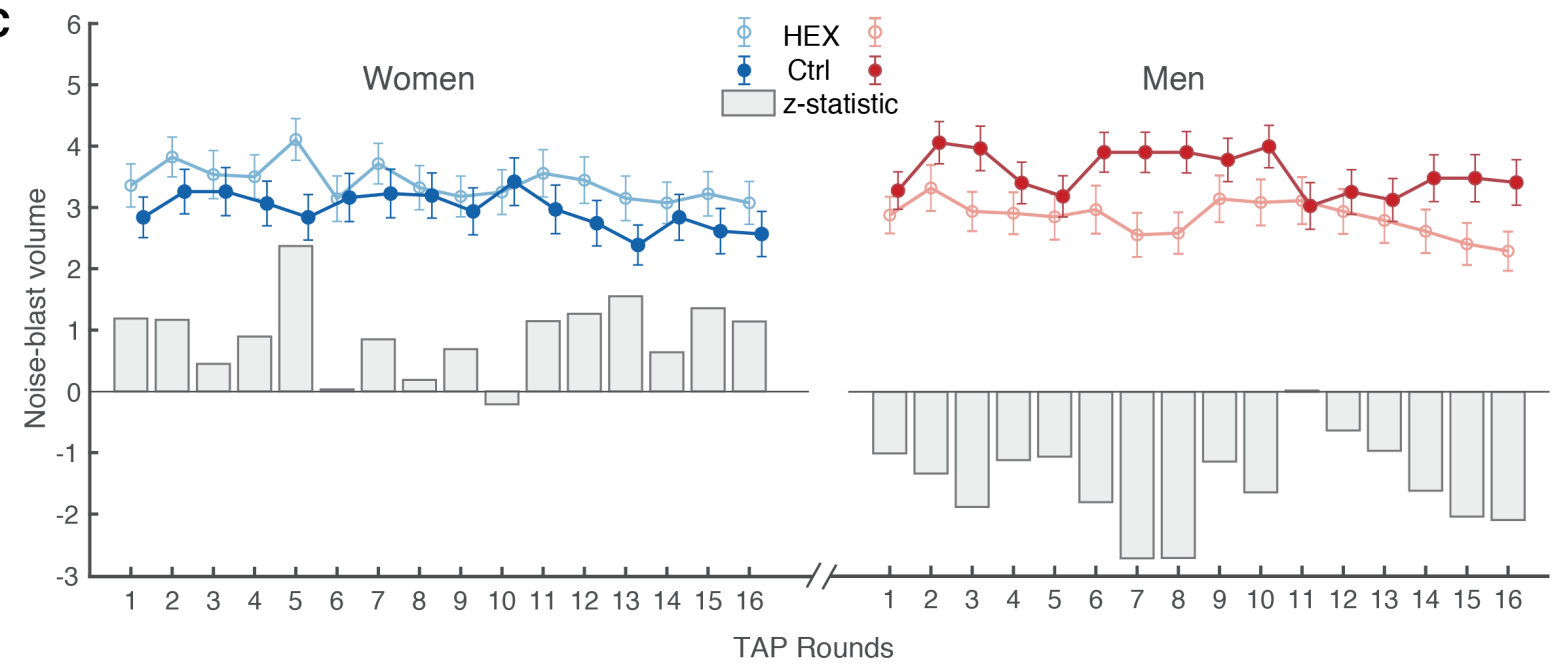

B

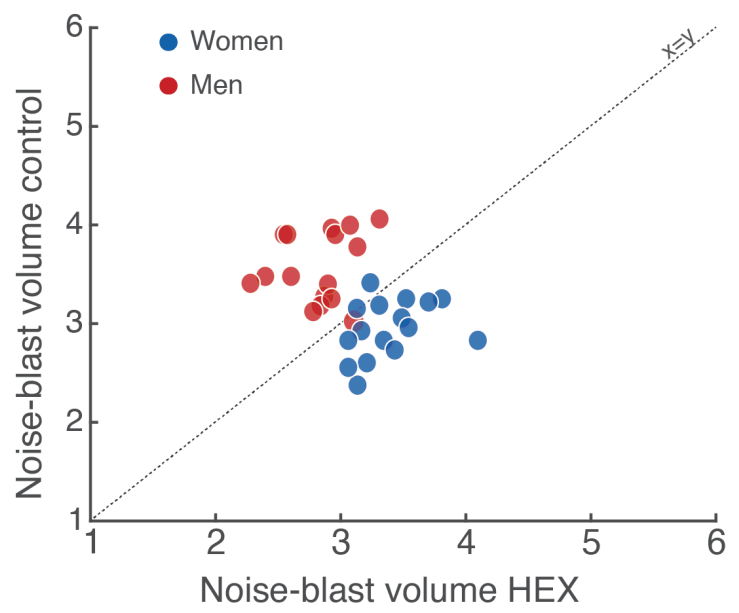

Noise-blast volume HEX

HEX

stic

Figure 3. Exposure to HEX modulated aggressive behaviour in a sex-dependent manner across participants

(A) Group-mean noise-blast volume. (B) Trial-wise mean noise-blast volume. Each dot is the mean of a trial (1-16) of all men (red) or women (blue) participants. The $Y$ axis reflects the trial mean during Control, and the $X$ axis reflects the trial mean during HEX. The dotted line is the unit slope line $(X=Y)$, such that data under the line reflect increased aggression in HEX and data above the line reflect decreased aggression in HEX. (C) A trial-by-trial depiction across participants. The solid dots reflect Control, the open circles reflect HEX and the grey bars reflect a trial-by-trial $Z$ test. Men: $n=67$ (34 Control). Women: $n=60$ (31 Control). All tests were two-tailed, all error bars represent SEM. ${ }^{*}=p<0.05, * * * *=p<0.0005$.

We next used functional magnetic resonance imaging ( $\mathrm{fMRI}$ ) in order to ask where and how in the brain does HEX impact aggression. We used the point subtraction aggression paradigm (PSAP), another well-established paradigm in aggression research that has been used in $\mathrm{fMRI}$ (38-40). It allows imaging of brain activity during provocation (having your money taken away from you) and aggression (subtracting money from others, yet not for monetary profit). In 
Mishor et al

order to provide for a more naturalistic aggressive output, we substituted the response buttons with fist-clench pressure sensors. Participants were misled to think that the responsedevice provides binary output alone, but in fact we measured fist-clench effort as a naturalistic implicit continuous measure of aggression extent. In support of this method, we observed a modest but significant correlation between measured aggression at baseline and basal aggressive tendencies as estimated by a standard aggression questionnaire (AGQ) (41) $(r=0.30, p=0.04)$ (Supplementary Figure 6). Such correlations typically emerge only in very large samples (42), so their emergence here underlies the power of the fist-clench PSAP (FCPSAP). In a within-subjects, double-blind design (Figure 1F), 49 participants (24 women, mean age $26.98 \pm 3.92$, range 19-36) completed an average of 134.39 ( \pm 15.33 ) trials (of all types), about half concurrent with HEX and about half with carrier (mineral oil) alone, counterbalanced for order (For full table of events, see Supplementary Table 1). 
A

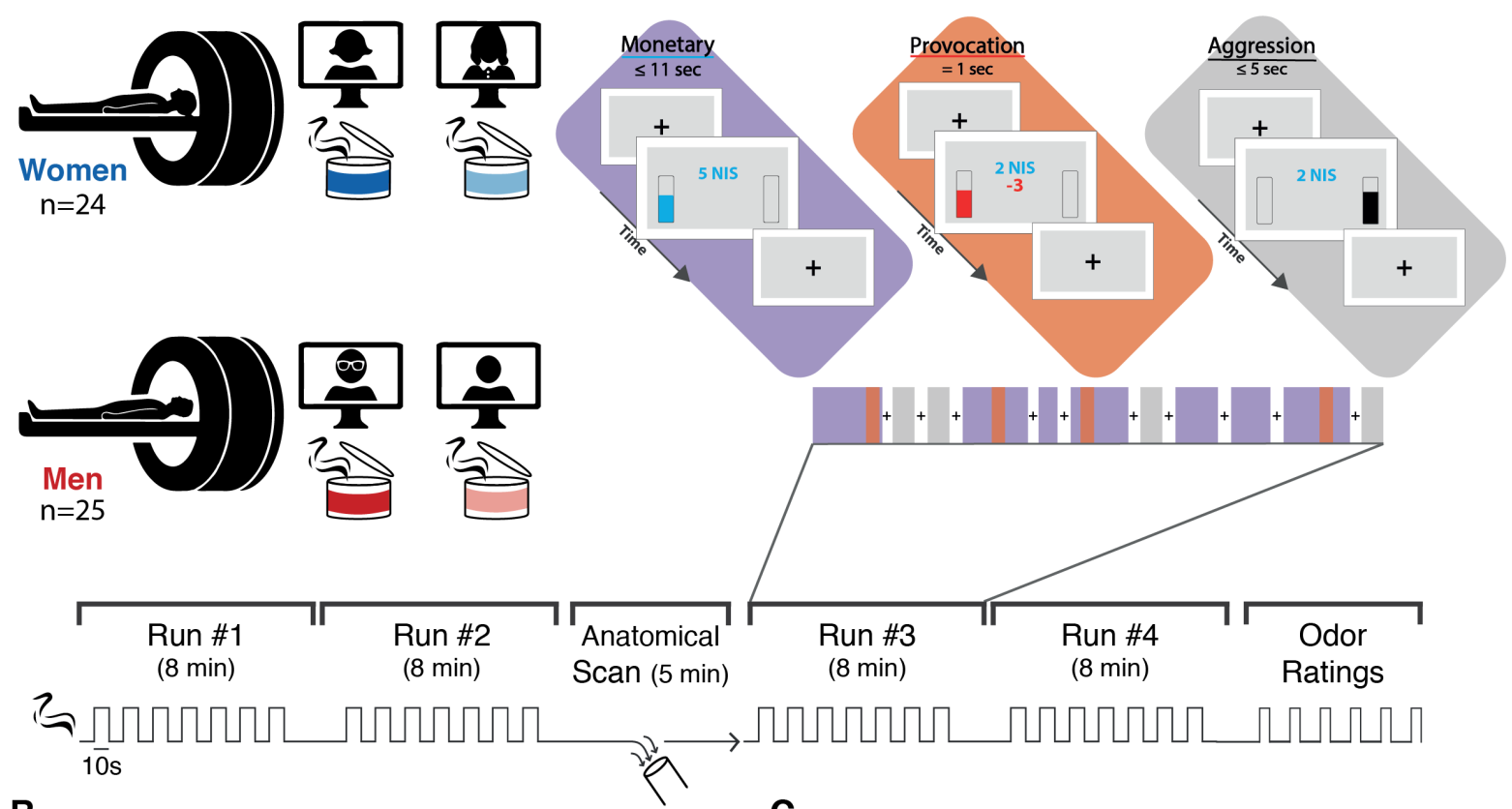

B
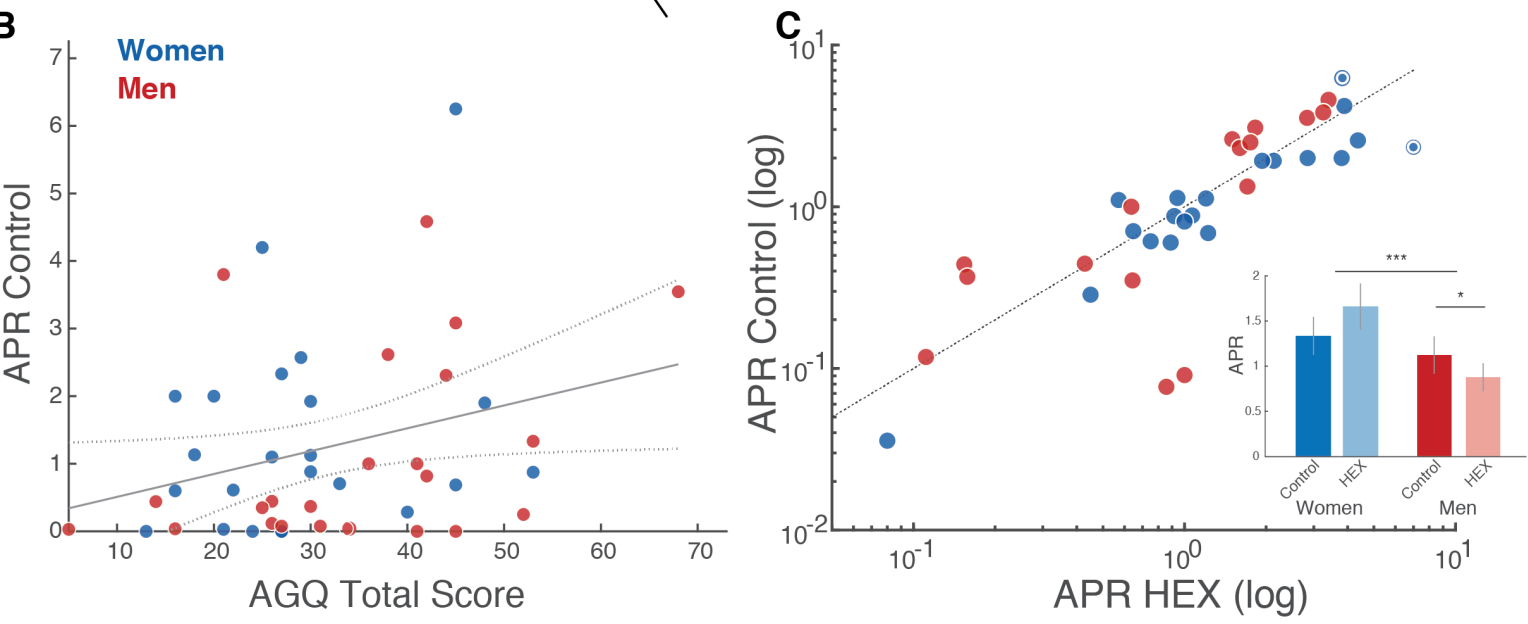

Figure 4. Exposure to HEX modulated aggressive behavior in a sex-dependent manner within participants

(A) In a within-subjects design, participants were exposed to an odorant (HEX and Control, counter-balanced for order), and played a game where their online partner stole their money occasionally (Provocation). During the game they could aggress against that same (nonexistent) person by deducting money from them (Aggression). (B) Correlation between basal levels of aggression as measured here (aggression/provocation ratio - APR in the control condition) and an aggression questionnaire. (C) Scatter plot of the APR under HEX and the APR in the control condition. Each dot is a participant, women (blue) and men (red). The scatter plot is summarized in a bar graph. Dotted circles represent outliers (>3 s.d.). All tests were two-tailed, all centres reflect mean, all error-bars reflect SEM. ${ }^{*}=p<0.05,{ }^{* * *}=p<0.005$. 
Mishor et al

\section{HEX again blocked aggression in men but triggered aggression in women}

To again ask whether the addition of HEX was associated with an explicit percept, participants used a visual-analogue scale to rate the stimuli along the primary dimensions of human olfactory perception; namely intensity and pleasantness (34) (Figure 1G, 1H). Because odorant ratings again did not distribute normally (Pleasantness: $W=0.98, p=0.001$, intensity: $\left.W=0.75, p=2.2^{*} 10^{-6}\right)$, we applied a linear mixed model with factors of Sex and Odor and random effects of participant. Analysis of the pleasantness ratings revealed a significant difference between the sexes in overall pleasantness in the FC-PSAP, no effect of Odor, and a marginally significant interaction $(\operatorname{Sex}: F(1,43)=8.07, p=0.007$, Odor: $F(2,175)=0.007, p=$ 0.94, Sex x Odor: $F(2,175)=3.08, p=0.05$ ). Post-hoc pairwise comparisons (Tukey adjustment for multiple comparisons) revealed that this difference arose from men's ratings of the odorless control. In other words, this reflected a basic sex-difference in reported valence (men Control-women Control: men mean VAS $=0.3 \pm 1.05$, women mean VAS $=-0.26 \pm 0.87$, $t(43)=3.09, p=0.04$. Men Control-women HEX: men mean VAS $=0.3 \pm 1.05$, women mean VAS $=-0.32 \pm 0.83, t(43)=3.12, p=0.04$. All other comparisons non-significant (all $t<2.78$, all $p>0.09$ ) (Figure 1I). To conclude, there were no differences within sex, and a marginal difference across sexes arising from men's ratings of the odorless control. The same model applied to intensity ratings reveled no main effect of sex, no interaction, but an effect of Odor (Sex: $F(1,43)=1.69, p=0.2$, Odor: $F(2,175)=3.88, p=0.02$, Sex $x$ Odor: $F(2,175)=0.41, p=$ 0.66). Post-hoc contrasts (Tukey adjustment for multiple comparisons) revealed that the intensity differences in the FC-PSAP arose from Control-Blank comparisons (blank mean VAS $=-0.17 \pm 0.88$, control mean VAS $=0.15 \pm 1.08, \mathrm{t}(175)=2.57, \mathrm{p}=0.03)$, but not Control-HEX comparisons (control mean VAS $=0.15 \pm 1.08$, HEX mean VAS $=-0.07 \pm-0.96, t(175)=2.14, p$ $=0.08$ ) (Figure $1 \mathrm{~J}$ ). In other words, mineral oil is detectable versus blank, but HEX had no impact on perception in this experiment.

In the PSAP, aggressive behaviour is typically reported as the aggression/provocation ratio (APR), namely the aggressive responses divided by the number of provocation events participants experienced. Higher APR values imply increased aggression. Using the withinsubjects FC-PSAP, we observed the same behavioral pattern we previously observed using the between-subjects TAP (Figure 4C). More specifically, a Kruskal-Wallis test on the delta 
bioRxiv preprint doi: https://doi.org/10.1101/2020 09 29.318287: this version posted December 14,2020 . The copyright holder for this preprint (which was not certified by peer review) is the author/funder, who has granted bioRxiv a license to display the preprint in perpetuity. It is made available under aCC-BY-NC-ND 4.0 International license.

Mishor et al

APR with a factor of Sex revealed a significant effect of Sex between odour conditions $(\chi 2=$ 9.24, $d f=1, p=0.002$, effect size $\eta^{2}=0.18$ ). This interaction reflected that HEX drove a significant reduction in aggression by men $(A P R$ control $=1.12 \pm 1.43$, APR HEX $=0.88 \pm 1.08$, Wilcoxon signed rank test: $Z=2.18, p=0.03$, effect size $r=0.45$ ), yet increased aggression by women [we note that two women were 3 standard deviations away from the APR mean, one of them from the Control APR mean, and the other from the HEX APR mean. Removing these two women from the analysis, the effect in women is APR control $=1.07 \pm 1.03$, APR HEX $=$ $1.32 \pm 1.31$, Wilcoxon signed rank test: $Z=2.15, p=0.03$, effect size $r=0.43$. If we do not remove these two outliers, we get APR control $=1.34 \pm 1.46, \mathrm{APR} H E X=1.66 \pm 1.76$, Wilcoxon signed rank test: $Z=1.90, p=0.06$, effect size $r=0.41$ ] (Figure $4 C$ ). Thus, in two consecutive experiments, one across-participants and one within-participants, HEX decreased aggression in men, but increased aggression in women.

\section{HEX increased activity in a brain area implicated in the perception of social cues}

To investigate the impact of HEX on brain response, we conducted a whole-brain voxel-wise statistical parametric map. The ANOVA contrast of provocation vs. baseline (monetary) revealed a typical salience network activation (Figure 5).

\section{$S$}

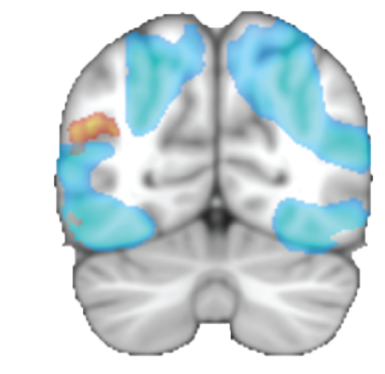

A
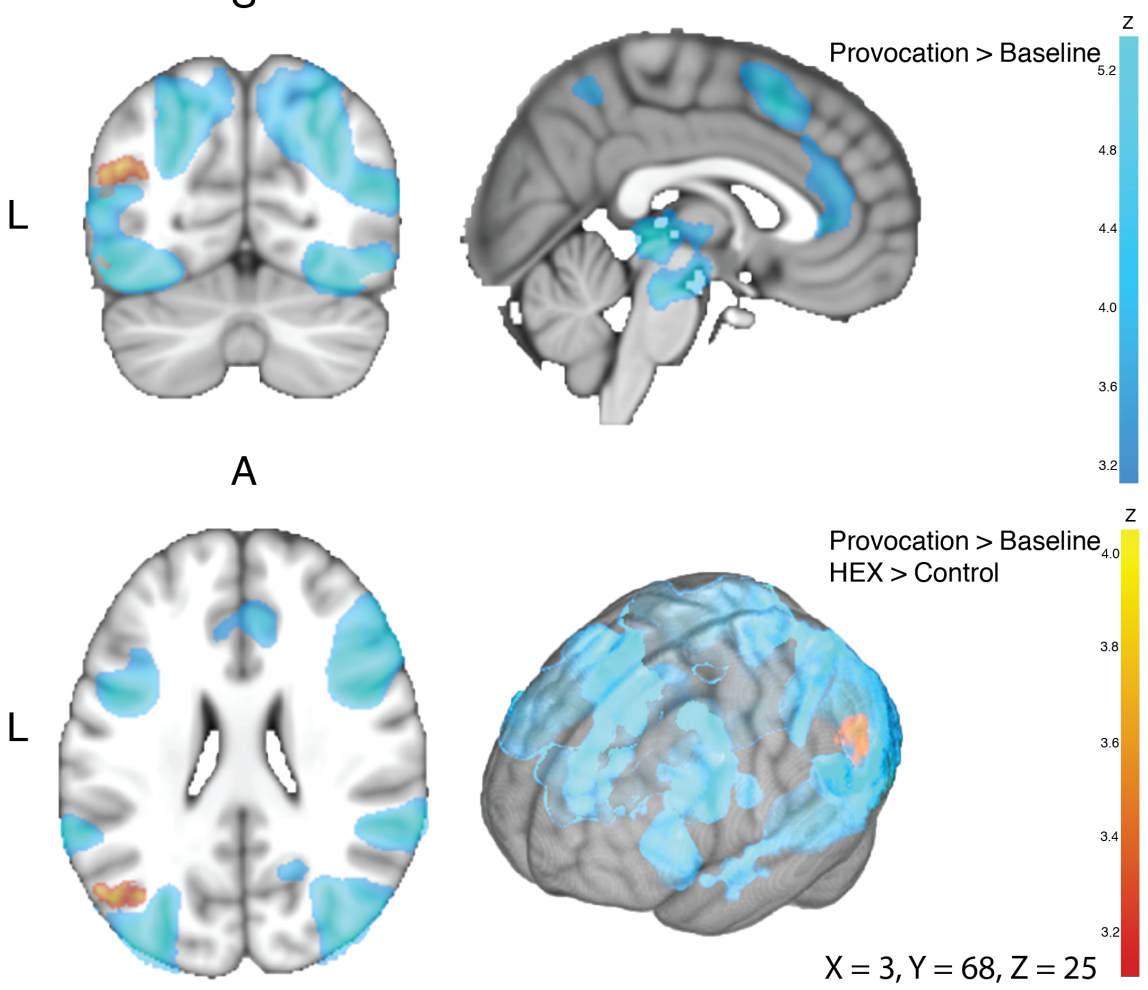
Mishor et al

\section{Figure 5. Provocation recruited an extensive brain network}

The group image ( $n=49$ ), in coronal (top left), sagittal (top right), axial (bottom left) and surface (bottom right) views. In all panels, shades of blue reflect the Provocation $>$ Baseline contrast, and shades of red reflect the Provocation > Baseline and HEX > Control contrast. In blue, provocation induced activity in the fusiform gyrus, OFC, Insula, Superior temporal gyrus, anterior cingulate cortex, inferior frontal gyrus, pre-SMA, precuneus, ventral tegmental area, periaqueductal grey area and thalamus (for full list, coordinates and peak activation, see Supplementary table 2). In red, HEX induced activation in left Angular gyrus.

In turn, the ANOVA contrast of provocation vs. baseline (monetary, see methods) with the added level of HEX vs. control in men and women revealed only one, but very pronounced significant activation in the left angular gyrus (AG), with no difference between men and women (Figure 5, Figure 6A). Although the statistical significance associated with this result is the mapping statistic ( $p<0.001$, corrected for multiple comparisons), in order to verify the directional drivers of this effect, we visualized the betas in the resultant left AG region of interest (Figure 6B, 6C). A two-tailed ANOVA applied to the beta values revealed a main effect of odour $(F(97)=6.79 p=0.01)$, but no effect of $\operatorname{sex}(F(1,97)=0, p=0.95)$ and no interaction $(F(1,97)=0.03, p=0.87)$. The main effect of odour reflected increased activation during provocation under HEX (mean beta (a.u.) HEX = 57.74 \pm 22.76 (SE), mean beta (a.u.) control = $-26.26 \pm 22.37$ ) (we reiterate that these statistics are merely to verify directionality, as the statistic associated with this finding remains the mapping statistic alone, namely $p<0.001$, corrected for multiple comparisons). In previous research, the left AG has been implicated in the perception of social cues (43), and was recruited by perception of contextual social cues (44). Moreover, the left AG has been associated with brain mechanisms of aggression (45), and it is hypoactive in aggressive individuals (46). In other words, the AG is part of a social network related to aggression, and here it was recruited by a social putative chemosignal. 
A

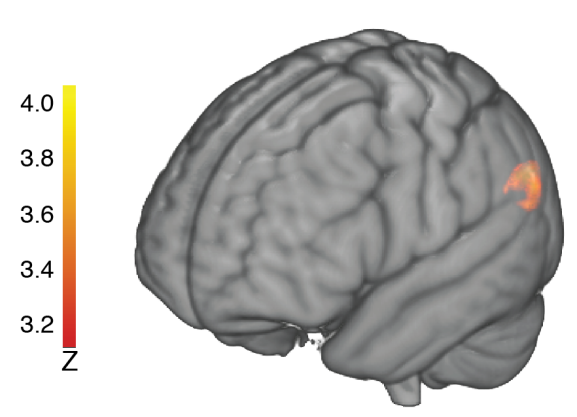

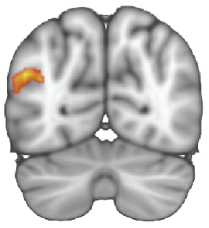

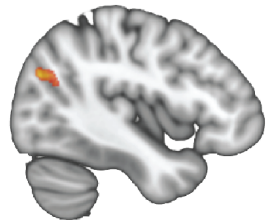

$X=-42$

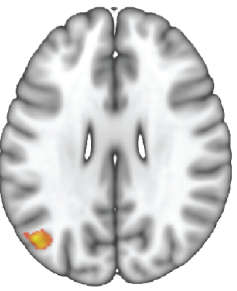

$Z=26$
B

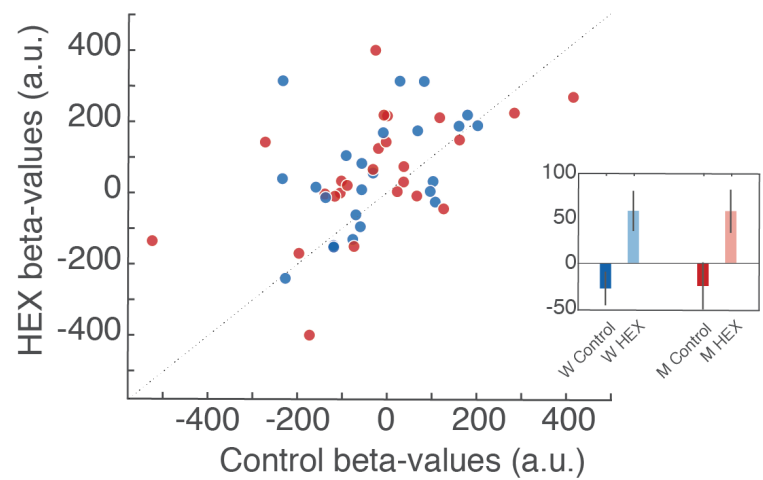

D

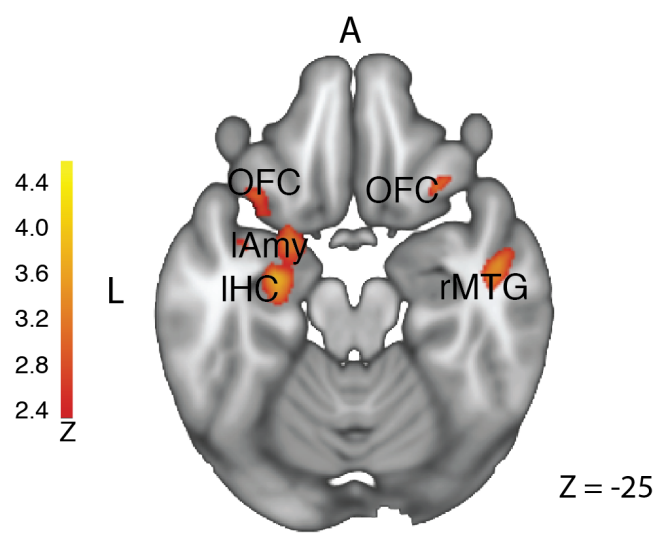

$\mathbf{E}$

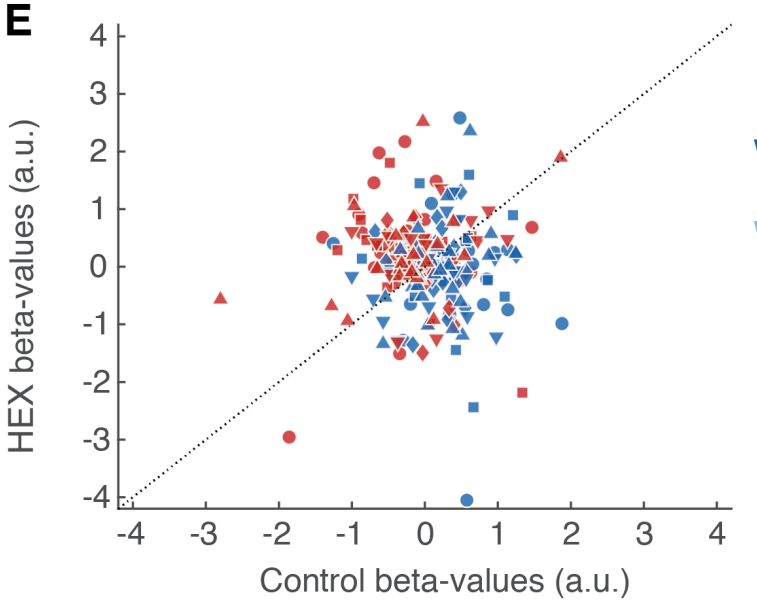

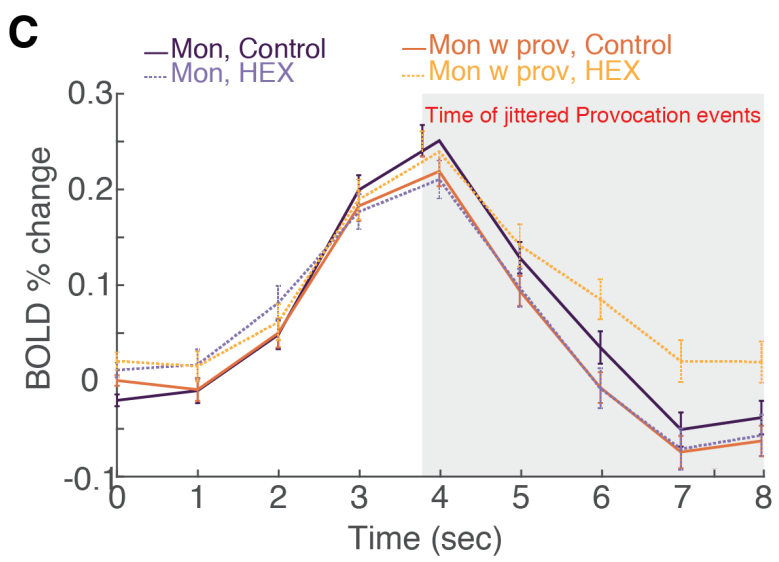

$\mathrm{S}$

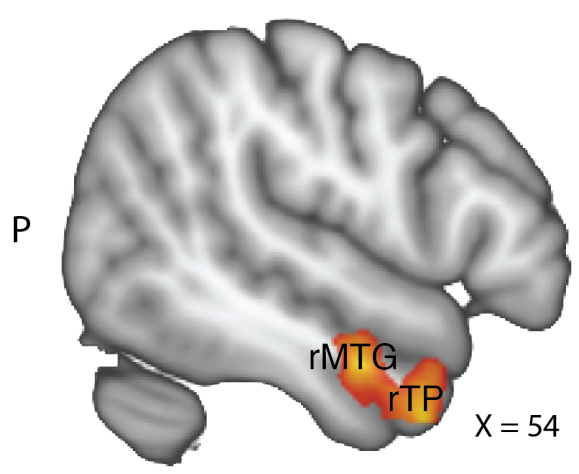

$\mathbf{F}$

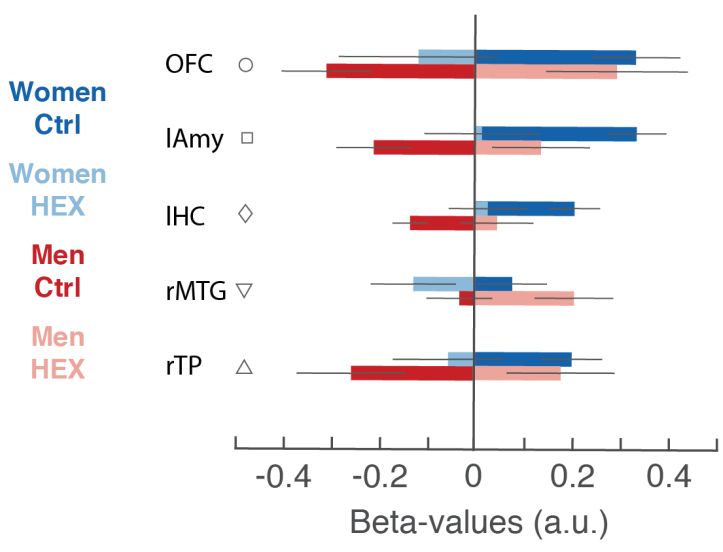

Figure 6. HEX drives sex-specific functional connectivity in the brain substrates of aggression

(A) A contrast ANOVA statistical parametric map depicting activity greater during provocation vs. baseline in HEX vs. Control ( $p<0.001$, corrected). 
(B) Beta-values extracted from the left angular gyrus. Each dot is a woman (blue) or man (red) participant. The $Y$ axis reflects that participant's mean Beta during HEX, and the $X$ axis reflects that participant's mean Beta during Control, during a Provocation. The dotted line is the unit slope line $(X=Y)$, such that data under the line reflect increased activation in Control and data above the line reflect increased activation in HEX. (C) The percent signal change in BOLD activity in the left angular gyrus ROI, depicted by condition. (D) A contrast statistical parametric map depicting functional connectivity with the left angular gyrus greater during provocation vs. baseline in HEX vs. Control in Men $>$ Women ( $<0.01$, corrected). OFC Orbitofrontal cortex, IAmy - left Amygdala, IHC - left Hippocampus, rMTG - right Medial temporal gyrus, rTP - right temporal pole. (E) Beta-values for functional connectivity with the angular gyrus. Each dot is a woman (blue) or man (red) participant, shape of dot is as depicted in panel F. (F) Bar graph of the beta values of functional connectivity of Provocation>Baseline, HEX>Control, Women>Men, for whole-brain connectivity analysis with the AG as a seed region.

\section{HEX modulated functional connectivity in brain networks of aggression}

To investigate how the left AG may be modulating aggression under HEX, we investigated its functional connectivity with the entire brain. We applied whole-brain psychophysiological interaction (PPI) analysis (47) using the left functional AG region of interest (ROI) as a seed. We observed a pronounced dissociation by sex, that mirrored behavior. More specifically, HEX significantly modified left AG functional connectivity with three brain regions: The right temporal pole (extended to the middle temporal gyrus), the left amygdala (extended to the left hippocampus) and bilateral lateral orbitofrontal cortex (Figure 6D). Although the statistic associated with these HEX-related changes in functional connectivity is the PPI mapping statistic $(p<0.01$ corrected for multiple comparisons [we note that the temporal pole result is stronger, surviving $p<0.001$ corrected]), to further explore directionality, we applied ANOVAs to the beta values of connectivity. We observed that under provocation, HEX systematically increased connectivity to these regions in men, but decreased it in women. This effect recurred in the right temporal pole $(F(1,97)=5.60, p=0.02$, Men: mean betavalues HEX $=0.18 \pm 0.16$, mean beta-values Control $=-0.25 \pm 0.16$. Women: mean beta-values HEX $=-0.05 \pm 0.16$, mean beta-values Control $=0.20 \pm 0.09$. In the left amygdala $(F(1,97)=$ 6.49, $\mathrm{p}$-value $=0.01$, Men: mean beta-values HEX $=0.14 \pm 0.14$, mean beta-values Control $=-$ $0.21 \pm 0.11$. Women: mean beta-values HEX $=0.01 \pm 0.17$, mean beta-values Control $=0.33 \pm$ 0.09. And in the lateral $\operatorname{OFC}(F(1,97)=8.40, p$-value $=0.005$, Men: mean beta-values HEX $=$ $0.29 \pm 0.21$, mean beta-values Control $=-0.31 \pm 0.13$. Women: mean beta-values HEX $=-0.12$ 
Mishor et al

\pm 0.24 , mean beta-values Control $=0.33 \pm 0.13$ (Figure $6 \mathrm{E}, 6 \mathrm{~F}$ ) (we reiterate that these statistics are merely to verify directionality, as the statistic associated with this finding remains the mapping statistic alone, namely $p<0.01$ corrected for multiple comparisons).

We observe that the temporal pole, amygdala, and OFC, are all parts of two brain-networks involved in aggressive behaviour; social and emotional evaluation and decision-making (48). Thus, our results imply that these regions may act in concert under the modulation of the AG, whereby increased functional connectivity with these regions is associated with reduced aggression (as in men), but decreased connectivity is associated with increased aggression (as in women). Finally, when looking at the shared change in FC for both women and men, we see an increase in FC for left motor areas: PMC and SMA (all participants were right handed, and used mostly the right hand for aggressive response). This is consistent with previous reports of increased activity in motor and pre-motor areas in response to provocations (15) (see Supplementary Figure 7).

\section{Discussion}

Impulsive aggression is a major factor in the human condition, yet how exactly aggression is triggered or blocked in the human brain remains unclear (15-17). Moreover, real-world human impulsive aggression is one of the most sexually dimorphic behaviours (49), yet what brain mechanisms underlie this dimorphism also remains unclear (17). In animals ranging from insects to rodents, aggression is sexually dimorphic at levels ranging from genes to cells $(50,51)$, and this dimorphism has been linked to the olfactory system $(4,52)$. Here we find the same in humans. We observed that a body-volatile, namely HEX, significantly decreased aggression in men, yet significantly increased it in women. This same molecule also increased brain activity in the AG, a cross-modal integrating hub involved in social cognition (53). In other words, in humans, like in rodents, a "social odour" activates the "social brain". Moreover, this same signal modulated functional connectivity between these substrates of social appraisal (AG) and a network previously associated with aggression. This included modulation of functional connectivity with the TP, an area similarly implicated in social appraisal (54) and aggression (55), and modulation of functional connectivity with the amygdala and OFC, namely substrates implicated in aggressive execution $(14,16,17)$. All this occurred in a sex-dependent manner consistent with behaviour: HEX increased connectivity 
Mishor et al

in men but decreased it in women. We would like to stress that in our analyses we did not first highlight the neural substrates of aggression and ask how they are impacted by HEX, but rather asked what brain networks are modulated by HEX, and this question happened to uncover the classic neural substrates of social appraisal and aggressive behaviour. We think this path of discovery significantly strengthens the idea that HEX is a human social chemosignal with relevance to aggression. Moreover, it provided for a behavioural and brain mechanistic depiction of human aggression and its sexual dimorphism: HEX may lead to increased or decreased aggression through increased or decreased control by the AG over the amygdala through a circuit involving the TP and OFC.

The sex dimorphism in our behavioural and brain results dovetails with previous findings obtained using functional brain imaging (56) to depict a level of functional brain sexdimorphism in response to social odours that is not matched by any other sensory stimulus that we are aware of. Human functional brain responses to basic auditory and visual cues are generally non-dissociable by sex (57), yet here we could use them alone to discriminate men from women at $79.6 \%$ accuracy. This begs the question: what behavioural setting could underlie selection for a body-odour that increases aggression in women but decreases it in men? Or in other words, what could be the ecological relevance of these results? In this respect, we call attention to the setting of infant rearing. Parents across cultures are encouraged to sniff their babies (58), an action that activates brain reward circuits in women (59). Although an initial analysis of baby-head volatiles did not report on HEX (60), in-depth follow-up by the same authors using GCXGC-MS uncovered hexadecanal at about $20 \%$ the level of heptanal, the most abundant aldehyde component (Mamiko Ozaki, personal communication). Moreover, we also note in this respect that infant rearing is the one social setting where humans have extensive exposure to conspecific faeces (61), a rich source of HEX (27). Our results imply that sniffing babies may increase aggression in mothers, but decrease aggression in fathers. Whereas maternal aggression has a direct positive impact on offspring survival in the animal world $(62,63)$, paternal aggression has a negative impact on offspring survival (64). This is because maternal aggression is typically directed at intruders, yet paternal aggression, and more-so non-paternal male aggression, is often directed at the offspring themselves (65-67). If babies had a mechanism at their disposal that increased 
Mishor et al

aggression in women but decreased it in men, this would likely increase their survival. Our results portray just such a mechanism.

Given all of the above, should we label HEX as a human pheromone? Sniffing human bodily secretions such as sweat and tears drives assorted behavioural and physiological effects (11, $68)$, and body-odours may reflect assorted emotional states $(69)$ including aggression $(18,19)$, but the identity of specific molecular components involved in human social chemosignaling has remained elusive (70). Here we identify one such component, namely HEX, whose effects can be seen as consistent with those of a mammalian pheromone (71). Previously, the steroidal molecules estratetraenol (EST) and androstadienone (AND) had been proposed as human pheromones, yet this labeling was often rejected, primarily because EST and AND don't clearly trigger or block behavior, nor do they have obvious ecological relevance $(72,73)$. Here HEX had a pronounced effect on behavior, and moreover, on the behavior of aggression, a domain dominated by pheromonal communication in most mammals (7). The notion of pheromonal communication was once considered dependent on a functional vomeronasal system, a system that humans may not possess (74). More recent views, however, blur this distinction, and highlight pheromonal communication through the main olfactory system as well (75-79). Despite these arguments for labelling HEX as a human pheromone, our results fall short of granting us that license. This is primarily because of one limitation: Although we demonstrated that the effects emerge following HEX and do not emerge following a control odour, and although the effects persisted despite a lack of percept associated with HEX, we nevertheless did not test against additional molecules also present in body-odour (e.g., the above-mentioned heptanal). In other words, it remains possible that the signal in question is "body-odour" (albeit subliminal), and not necessarily HEX alone. We submit that this does not take away from the fundamental findings we present, namely that a social chemosignal can trigger or block aggression, the pronounced sexual dimorphism in this cascade, and identification of the brain substrates involved, but it does raise the possibility that HEX is not unique in this, and that other body-volatiles may have similar effects. This issue remains to be explored, and currently prevents us from labelling HEX alone as a human pheromone. Beyond this, we would like to acknowledge several limitations: First, regarding our imaging results, we reiterate that correlation is not causation. We indeed identify a brain pattern associated with HEX, and it is tempting to suggest that this pattern is responsible for the 
Mishor et al

observed effects, yet this can only be proven by experiments where the brain mechanism is perturbed, experiments that are very difficult to conduct in human participants. Second, we also acknowledge that our suggested ecological relevance in infant rearing was not directly tested in this study. Although we think it is a plausible hypothesis, it remains to be experimentally verified, and here serves only as an example of possible ecological relevance for our results.

Despite the above limitations, we conclude in stating that sniffing the body-odour-constituent HEX blocks aggression in men but triggers aggression in women. HEX may exert its effects by modulating functional connectivity between the brain substrates of social appraisal and the brain substrates of aggressive execution. This places chemosignaling at the mechanistic heart of human aggression, and poses but one added example to the rapidly growing body of evidence implicating social chemosignaling as a major, albeit mostly subconscious, power in human behaviour.

\section{Acknowledgments}

This work was funded by an ERC AdG grant (SocioSmell 670798) awarded to Noam Sobel. We thank Macia Buades-Rotger, Ulrike Kramer, Katja Bertsch, Jeanette Mumford, Yaara Yeshurun and Anat Arzi for their valuable guidance.

\section{Author Contributions}

N.S, H.B, J.S and E.M. conceptualized the idea. E.M. planned and created the experiments, acquired, analyzed, interpreted and visualized the data. D.A. acquired men data in the TAP. E.M and T.W. analyzed imaging data, D.H., A.W. and E.M created the pressure sensor apparatus. E.L. and L.G. handled the olfactometer. E.M., D.K. and R.Z. performed the ELISA. A.R. wrote code for the TAP. R.W., T.S., Y.E.S., S.A, L.R., N.R., A.R., L.G. and E.L. helped run participants. E.M. and N.S. wrote the manuscript.

\section{Declaration of Interests}

The Weizmann Institute of Science has filed for patent on the use of HEX to modify human behaviour and mood. 
Mishor et al

\section{Methods}

\section{Data availability}

All data of this manuscript will be publically available on publication. All the behavioural data are already available on Mendeley at doi:10.17632/6nkh8j8733.1. All brain imaging data will be uploaded to OpenNeuro on acceptance.

\section{Code availability}

All experiment and analysis codes will be uploaded to https://gitlab.com/worg wis/evaaggression

\section{Taylor Aggression Paradigm (TAP)}

Participants: To estimate the number of needed participants we conducted a power analysis. We used the effect size obtained with aggression-related chemosignals (18) and using G*Power software (80) estimated, at alpha $=0.05$ and $80 \%$ power, a necessary sample of at least 105 participants. Based on this, a total of 127 participants (67 men, mean age $25.48 \pm$ 3.46, range 21-34) were recruited to a modified Taylor aggression paradigm (TAP) (30-33) after providing written informed consent to procedures that were approved by the Weizmann Institute Institutional Review Board (IRB). Participants were recruited using ads, and had no history of psychiatric drug use or any neurological or nasal conditions.

Odorant delivery: We used methods we have applied extensively in the past $(29,81)$. In an across-subjects double-blind design, half of the participants were exposed to carrier alone (10\% eugenol, 100ul, diluted in Propylene Glycol) (control), and half to HEX masked in $10 \%$ eugenol (100ul, $0.083 \mathrm{M})$. In brief, before commencement of the TAP, participants were first exposed to the odorant. Exposure started with 11 rating trials (inter-trial-interval $=25$ seconds). On each trial the participant sniffed from an unmarked odorant-jar for 3 seconds, and then rated pleasantness, intensity and familiarity of the odorant along a visual-analogue scale. After the ratings session, an adhesive pad containing 30ul of the odorant was pasted onto the upper lip of the participant for continued exposure throughout the experiment. Participants and experimenters were of the same sex (three alternating men experimenters, 
Mishor et al

four alternating women experimenters), and it was also noted to participants that their unseen game-partner is of the same sex.

Provocation phase: Provocation is a necessary catalyst for aggression in laboratory studies (82). To this end, participants were first antagonized towards a purported game-partner (Figure 1) in a form of the ultimatum game (83). In five rounds, participants were asked to distribute an amount of money ( 9\$) between themselves and their fictitious game-partner. If they were to reach agreement, the distributed amount would be added to their earnings in the experiment. However, the interaction was rigged such that the fictitious game-partner refused any fair distribution, and agreed only to distributions where he/she received almost the entire amount (>8\$). In order to maintain reliability, the time it took the purported gamepartner to respond to offers was U-shaped, shorter for low and high amounts. The apparent egregious behaviour of the fictitious game-partner served as the aggression provoking mechanism.

Aggression discharge phase: After the provoking phase, participants engaged in a modified Taylor aggression paradigm. Participants were again misled to believe that they are playing against the same partner (from the provocation phase). This time, in a reaction-time task where they compete to identify a change in shape of a target. If they were faster to identify, they could blast their opponent with an unpleasant noise (noise-blast) at a volume of their choosing. If their game-partner was faster, they would endure a noise-blast induced by their game-partner. Determining which of the participants was faster was done using a responsive calculation. This assured that participants will induce noise-blasts 16 out of 27 rounds, yet with respect to their actual reaction times. Additionally, this served the purpose of, again, maintaining reliability of the experimental setup. Noise-blasts were induced using a button box with 6 buttons labelled with informative faces (modified from a pain scale for children (84) (Figure 2A). The faces portrayed the intensity of the noise-blast, from mildest (1) to most severe (6). Noise-blasts that participants heard were randomized in length ( $\sim$ seconds) and volume (Mean $=90 \mathrm{db}$, coming from a speaker in front of them). 
Mishor et al

Questionnaires: Prior to any experimental manipulation, self-reported mood was measured using a commonly applied 17-item mood questionnaire (85) (Supplementary Figure 1). At the end of the experiment, we asked participants to rate, using a VAS, general questions probing their social evaluation and willingness to interact with their game-partner (Supplementary Figure 8; Supplementary Figure 9). Additionally, participants completed the autism quotient questionnaire (AQ) (86), State anxiety questionnaire (STAI) (87) and the Buss and Perry aggression questionnaire (AGQ) (41) (Supplementary Figure 6).

Statistical analysis: Odor ratings were standardized with respect to the entire sample mean and s.d. Odour ratings did not distribute normally (Pleasantness TAP: Shapiro-Wilk W=0.98, $p=0.001$, intensity TAP: $W=0.96, p=0.002$. Pleasantness FC-PSAP: $W=0.98, p=0.001$, intensity FC-PSAP: $\left.\mathrm{W}=0.75, \mathrm{p}=2.2^{*} 10^{-6}\right)$. Therefore, we applied a linear mixed model with factors of Sex and Odor, and random effect of participant. Noise-blasts were standardized with respect to the entire sample mean and s.d. Noise-blast volume was entered into a repeated-measures ordinal logistic regression analysis (Cumulative Link Mixed Model fitted with the Laplace approximation) with factors of Odorant (HEX/Control) and Sex (men/women), and random effects of Participant. We further explored the data using the trial-by-trial mean for each group (16 separate noise-blast trials for each participant). In order to quantify this, we first conducted a Mann-Whitney test to compare between groups within trial. Then, an independent t-test on the trial-by-trial z-values. For further validation, we used a random permutation test. We shuffled the odour conditions of the real data and conducted a t-test on the shuffled z-distributions. We repeated the procedure 10,000 times and created a distribution of the t-statistics, resulting from statistical tests on the shuffled z-distributions. For reaction times, we first z-scored with respect to the entire group's mean and s.d. RT's did not distribute normally (Shapiro-Wilk: $W=0.49, p<2.2^{-16}$ ). Therefore, we applied a linearmixed model with factors of Odour, Sex, Round, and participant as a random effect. For the exit questionnaire: Responses were z-scores to each Participant's range. Self-reported mood was analysed using a linear-mixed model with factors of gender, odour, and question, and participant as a random effect.

Exclusions: Participants: Three participants were excluded from the TAP analysis due to technical faults of the acquisition system. Five participants did not answer the exit 
Mishor et al

questionnaire, but were included in all other analyses. Trials: In the reaction time analysis we removed outliers of $>3$ s.d. This resulted in removal of 99 trials out of 3456 . This constitutes $2.86 \%$ of total trials, and $18.5 \%$ of trials in the one participant with most exclusions.

\section{Fist-Clench Point Subtraction Aggression Paradigm (FC-PSAP)}

Participants: To estimate the number of needed participants we conducted a power analysis. We used the effect size obtained in the TAP to estimate group size using G*Power software. This implied that at alpha $=0.05$ and $80 \%$ power we need to test a sample of 50 participants. Based on this, a total of 58 participants, of which 49 were included in final analyses (24 women, mean age $26.98 \pm 3.92$, range 19-36) participated in a modified Point Subtraction Aggression Paradigm (PSAP), after providing written informed consent to procedures that were approved by the Wolfson Hospital Helsinki Committee, and the Weizmann Institute Institutional Review Board (IRB). Participants were recruited using ads, and had no history of psychiatric drug use or any neurological or nasal conditions. All participants were righthanded.

FC-PSAP: Participants were told that their goal is to earn as much money as possible, and that we are interested in the dynamics of the interaction between them and the other participants. Participants actuated the PSAP using fist-clench (FC) activated pressure sensors (MR-compatible). The fist-clench devices were built in-house. They constituted a rubber ball in the hands of the participant, that was linked via $1 / 4$-inch Tygon tubing to a pressure sensor (all Sensors, 1INCH-Dx-4V-MINI). The sensors were powered at 5V (DAQ NI USB-6008), and the resultant signal was read using custom software written in MATLAB (code available at GitLab https://gitlab.com/worg wis/eva-aggression) and LabChart7 software (ADInstruments, New South Wales, Australia). The software includes electrical noise removal, and calculation of a threshold. In the FC-PSAP, participants pressed both sensors simultaneously to earn money (monetary event), using one sensor, no matter which, they deducted money from the other participant (aggression). Provocation onsets were prerandomized, and held constant across participants. The experimental timeline was such that the first MRI head scout was performed while participants calibrated the pressure sensors using alternating strong and weak presses, repeated three times. This served both the 
purpose of adapting the system to individual differences in pressure applied and also having the participants accustomed to the use of the FC pressure sensors (FCPS). Ensuing run duration was 8 minutes. After completing two runs with one odor condition, an anatomical scan was obtained. During the duration of the anatomical scan (5 minutes), air in the bore was high throughput vacuumed. Before starting the third run, participants were told that their (fictitious) game-partner was replaced, and now they are interacting with a new participant. This was done to minimize transferring of emotions, strategies etc. between odor conditions.

Odorant delivery in the MRI: Odorants were delivered using a computer-controlled airdilution olfactometer of the type we have used extensively in the past $(81,88)$. Here we also introduced one modification whereby rather than using a nose-mask for delivery, we converted the entire head-coil into a controlled olfactory microenvironment. The head-coil was first enclosed with clear Teflon-coated Plexiglas. The olfactometer Teflon nosel was placed $\sim 10 \mathrm{~cm}$ anterior to the nose, and produced a constant flow of olfactometer air (1.5 Ipm) that carried embedded $10 \mathrm{~s}$ pulses of odorant (HEX or control). A powerful 2-inch vacuum hose was connected to the back of the head coil, generating a constant directionalflow environment from the nosel in front of the nose, passed the nose, and to the back of the head-coil for removal. Nasal airflow was constantly precisely monitored using a nasal cannula linked to spirometer (ML141, ADInstruments), and instrumentation amplifier (Power-Lab 16SP, ADInstruments). Since Hexadecanal at this concentration and mineral oil have no perceivable odor, we validated precise olfactometer timing with a photoionization detector (PID) (RAE systems, Model ppbRAE 3000). In each experiment, there was a total of 4 runs, 2 with HEX (CAS\# 629-80-1, $0.083 \mathrm{M}$, supplied by $\mathrm{TCl}$ and Cayman), and two with control (mineral oil alone, CAS\# 8042-47-5, Sigma-Aldrich). Order of conditions was counterbalances, and the given condition was unknown to participants or experimenters. Only two out of 58 participants reported that they smelled any odor during the experiment. Odor ratings were completed inside the scanner, after completion of the FC-PSAP. At the end of the experiment participants completed an exit-questionnaire probing their thoughts, strategies and attitudes towards their game partners, a general demographics questionnaire, $A G Q, A Q$, and STAI.

MRI Data acquisition: MRI Scanning was performed on a 3 Tesla Siemens MAGNETOM Prisma scanner, using a 32-channel head coil. Functional data were collected using a T2*-weighted 
bioRxiv preprint doi: https://doi.org/10.1101/2020.09.29.318287; this version posted December 14,2020 . The copyright holder for this preprint (which was not certified by peer review) is the author/funder, who has granted bioRxiv a license to display the preprint in perpetuity. It is made available under aCC-BY-NC-ND 4.0 International license.

Mishor et al

gradient-echo planar imaging sequence. In each run there were 240 repetitions comprising 56 slices, axial slices tilted $15^{\circ}$ toward the AC-PC plane (TR=2000 ms, TE $=30 \mathrm{~ms}$, flip angle $=75^{\circ}$, field of view $240 \times 240 \mathrm{~mm}$, matrix size $96 \times 96$, slice thickness of $2.5 \mathrm{~mm}$ with no gap, $2.5 \times 2.5$ $\mathrm{mm}^{2}$ in-plane resolution), covering whole brain. Anatomical images were acquired at 3D T1weighted magnetization prepared rapid gradient-echo sequence at high resolution $(1 \times 1 \times 1$ $\mathrm{mm}^{3}$ voxel, $\mathrm{TR}=2300 \mathrm{~ms}, \mathrm{TE}=2.98 \mathrm{~ms}$, inversion time $=900 \mathrm{~ms}$, flip angle $=9^{\circ}$ ).

fMRI data analysis: Neuroimaging data was analyzed using FSL (5.0.9), FEAT (v6.00), SPM12 (for PPI analysis) and MATLAB (R2017b, R2018b). FMRI data processing was carried out using FEAT (FMRI Expert Analysis Tool) Version 6.00, part of FSL (FMRIB's Software Library, www.fmrib.ox.ac.uk/fsl). Registration of the functional data to the high-resolution structural image was carried out using the boundary-based registration algorithm (89). Registration of the high resolution structural to standard space images was carried out using FLIRT $(90,91)$ and was then further refined using FNIRT nonlinear registration $(92,93)$. The following prestatistics processing was applied; motion correction using MCFLIRT (91); non-brain removal using BET (94); spatial smoothing using a Gaussian kernel of FWHM 6mm; grand-mean intensity normalisation of the entire $4 D$ dataset by a single multiplicative factor; high-pass temporal filtering (Gaussian-weighted least-squares straight line fitting, with sigma $=62.5$ sec). For each participant, a first-level general linear model included three regressors of: monetary ( $<11 \mathrm{~s})$, provocation (=1 s) and aggressive events $(<5 \mathrm{~s})$, onset and offset were determined according to the visual stimuli presented to participants. Only offset times of monetary events dependent on participants' behavior. Additionally, we regressed out failed events ('none' regressor), temporal derivatives, and modeled out single TR's with excessive motion according to frame-wise displacement $>0.9$. The signal was convolved with doublegamma Hemodynamic response function (HRF). First, we modeled each run separately for each participant ( 4 in total), then combined the runs, adding the odor present for each run (two runs exposed to HEX, two to control, counterbalanced). Then we grouped the data, while adding a parametric modulation of participants' sex. Time-series statistical analysis was carried out using FILM with local autocorrelation correction (95). The second-level analysis, which separated different odor condition runs and combined same odor runs, was carried out using a fixed effects model, by forcing the random effects variance to zero in FLAME (FMRIB's Local Analysis of Mixed Effects) (96), (97), (98). The group-level analysis, which added 
Mishor et al

participants' sex and averaged across all group, was carried out using FLAME (FMRIB's Local Analysis of Mixed Effects) stage 1 (96), (97), (98). Z (Gaussianised T/F) statistic images were thresholded using clusters determined by $Z>2.3$ and a (corrected) cluster significance threshold of $P=0.05$ (99). Analysis focused on provocation events for several reasons. First, Since the response in question is impulsive aggression, the moment of the provocation is the moment when the reactive spontaneous response happens, this is, by definition, the provocation event. Additionally, provocation events were well defined temporally, and most reproducible across participants. Finally, some participants did not have enough aggressive responses to allow analysis. Moreover, some participants had aggressive responses only in one odor condition and not the other, which severely skews the analysis. In the manuscript we report a contrast of Provocation > Baseline. Notably, due to the particular design in which provocation events occurred only during monetary events, even though technically the contrast is defined as provocation Vs. baseline, it is de facto a Provocation > Monetary contrast that we are estimating. We refer to it throughout the text as Provocation > Baseline. Due to this design, we also refrain from calculating the $\%$ change, and refer to betas as a measure of activation.

PPI analysis: To explore functional connectivity with the AG during provocation we conducted a whole-brain psychophysiological interactions analysis (PPI) (100), using the angular gyrus (AG) region of interest (ROI) as a seed region. The AG ROI was functionally defined from the GLM analysis of the group-level. We conducted a whole-brain PPI analysis using FSL, with the first regressor being the provocation events regressor (psychological regressor), the second was the time-course of the AG ROI (physiological regressor), and the third was the PPI regressor of the convoluted response (interaction regressor) generated using SPM12. Other regressors were the task regressors as in the GLM model (monetary, aggression, none and motion according to frame-wise displacement $>0.9$ ). The following pre-statistics processing was applied; grand-mean intensity normalization of the entire 4D dataset by a single multiplicative factor. Time-series statistical analysis was carried out using FILM (95). Secondlevel analysis, in which we contrasted the odor conditions and combined same-odor runs, was carried out using a fixed effects model, by forcing the random effects variance to zero in FLAME (FMRIB's Local Analysis of Mixed Effects) (96), (97), (98). Group-level analysis, in which we added the sex parameter and averaged across participants, was carried out using FLAME 
Mishor et al

(FMRIB's Local Analysis of Mixed Effects) stage 1 (96), (97), (98). Z (Gaussianised T/F) statistic images were thresholded using clusters determined by $Z>2.58$ and a (corrected) cluster significance threshold of $\mathrm{P}=0.05$ (99).

Exclusions: One participant reported she did not believe the purported participant was real, and was therefore excluded from further analyses. Additional participants were excluded from analyses due to technical problems ( $n=5), A Q$ score above 32 ( $n=1$ women) (in accordance with (86)), or excessive head-movements during the scan $(n=1)$, retaining 24 women and 25 men in all reported analyses.

Data analysis tools: All analyses and statistical analyses were done using MATLAB R2018a and R2018b (The MathWorks, Inc.), FSL 5.0.9 (101), FEAT 6.00 (95, 97), SPM12 (102), and R 3.6.1 (103) (packages: readxl version 1.3.1, tidyverse 1.2.1, Hmisc version 4.3-0, plyr version 1.8.4, RColorBrewer version 1.1-2, reshape2, Ime4 version 1.1-21, emmeans version 1.3.5, nlme version 3.1-140, pwr version 1.3-0, DescTools version 0.99.35). 
Mishor et al

\section{References}

1. C.-t. Lee, D. W. Ingersoll, in Hormones and Aggressive Behavior, B. B. Svare, Ed. (Springer US, Boston, MA, 1983), pp. 373-392.

2. D. Muller-Schwarze, Chemical signals in vertebrates. (Springer Science \& Business Media, 2012).

3. E. B. Keverne, Mammalian pheromones: from genes to behaviour. Current biology 12, R807-R809 (2002).

4. M. L. Cheal, R. L. Sprott, Social olfaction: A review of the role of olfaction in a variety of animal behaviors. Psychological Reports 29, 195-243 (1971).

5. R. Mykytowycz, B. Goodrich, Skin glands as organs of communication in mammals. Journal of Investigative Dermatology 62, 124-131 (1974).

6. M. Novotny, S. Harvey, B. Jemiolo, J. Alberts, Synthetic pheromones that promote inter-male aggression in mice. Proceedings of the National Academy of Sciences 82, 2059-2061 (1985).

7. P. Chamero et al., Identification of protein pheromones that promote aggressive behaviour. Nature 450, 899 (2007).

8. J. J. McGlone, S. E. Curtis, E. M. Banks, Evidence for aggression-modulating pheromones in prepuberal pigs. Behavioral and neural biology 47, 27-39 (1987).

9. D. M. Ferrero et al., A juvenile mouse pheromone inhibits sexual behaviour through the vomeronasal system. Nature 502, 368-371 (2013).

10. U. Shanas, J. Terkel, Mole-rat harderian gland secretions inhibit aggression. Animal behaviour 54, 1255-1263 (1997).

11. S. Gelstein et al., Human tears contain a chemosignal. Science 331, 226-230 (2011).

12. T. J. Oh, M. Y. Kim, K. S. Park, Y. M. Cho, Effects of chemosignals from sad tears and postprandial plasma on appetite and food intake in humans. PLoS One 7, e42352 (2012).

13. N. Chernyak, K. Leimgruber, Y. Dunham, J. Hu, P. Blake, Paying back those who harmed us but not those who helped us: Direct negative reciprocity precedes direct positive reciprocity in early development. (2019).

14. R. J. Nelson, B. C. Trainor, Neural mechanisms of aggression. Nature Reviews Neuroscience 8, 536-546 (2007).

15. J. R. Fanning, S. Keedy, M. E. Berman, R. Lee, E. F. Coccaro, Neural correlates of aggressive behavior in real time: a review of $\mathrm{fMRI}$ studies of laboratory reactive aggression. Current behavioral neuroscience reports 4, 138-150 (2017).

16. L. J. Siever, Neurobiology of aggression and violence. American Journal of Psychiatry 165, 429-442 (2008).

17. M. E. Flanigan, S. J. Russo, Recent advances in the study of aggression. Neuropsychopharmacology 44, 241 (2019).

18. S. Mutic, V. Parma, Y. F. Brünner, J. Freiherr, You smell dangerous: communicating fight responses through human chemosignals of aggression. Chemical senses 41, 3543 (2015).

19. S. Mutic, Y. F. Brünner, R. Rodriguez-Raecke, M. Wiesmann, J. Freiherr, Chemosensory danger detection in the human brain: Body odor communicating aggression modulates limbic system activation. Neuropsychologia 99, 187-198 (2017). 
20. B. Klein et al., Activation of the mouse odorant receptor 37 subsystem coincides with a reduction of novel environment-induced activity within the paraventricular nucleus of the hypothalamus. European Journal of Neuroscience 41, 793-801 (2015).

21. R. Tirindelli, M. Dibattista, S. Pifferi, A. Menini, From pheromones to behavior. Physiological reviews 89, 921-956 (2009).

22. C. M. Nieberding et al., Cracking the olfactory code of a butterfly: the scent of ageing. Ecology letters 15, 415-424 (2012).

23. M. Tsunoda et al., Identification of an Intra-and Inter-specific Tear Protein Signal in Rodents. Current Biology 28, 1213-1223. e1216 (2018).

24. R. Hoppe, T. D. Lambert, P. B. Samollow, H. Breer, J. Strotmann, Evolution of the "OR37" subfamily of olfactory receptors: a cross-species comparison. Journal of molecular evolution 62, 460-472 (2006).

25. C. Verbeurgt et al., Profiling of olfactory receptor gene expression in whole human olfactory mucosa. PloS one 9, e96333 (2014).

26. J. D. Mainland et al., The missense of smell: functional variability in the human odorant receptor repertoire. Nature neuroscience 17, 114 (2014).

27. B. de Lacy Costello et al., A review of the volatiles from the healthy human body. Journal of breath research 8, 014001 (2014).

28. S. K. Jha, N. Marina, C. Liu, K. Hayashi, Human body odor discrimination by GC-MS spectra data mining. Analytical Methods 7, 9549-9561 (2015).

29. Y. Endevelt-Shapira et al., Altered responses to social chemosignals in autism spectrum disorder. Nature neuroscience 21, 111-119 (2018).

30. S. P. Taylor, Aggressive behavior and physiological arousal as a function of provocation and the tendency to inhibit aggression 1 . Journal of personality 35, 297310 (1967).

31. M. Elson, M. R. Mohseni, J. Breuer, M. Scharkow, T. Quandt, Press CRTT to measure aggressive behavior: The unstandardized use of the competitive reaction time task in aggression research. Psychological Assessment 26, 419 (2014).

32. P. R. Giancola, D. J. Parrott, Further evidence for the validity of the Taylor aggression paradigm. Aggressive Behavior: Official Journal of the International Society for Research on Aggression 34, 214-229 (2008).

33. P. R. Giancola, S. T. Chermack, Construct validity of laboratory aggression paradigms: A response to Tedeschi and Quigley (1996). Aggression and Violent Behavior 3, 237253 (1998).

34. R. M. Khan et al., Predicting odor pleasantness from odorant structure: pleasantness as a reflection of the physical world. Journal of Neuroscience 27, 10015-10023 (2007).

35. W. Giith, On ultimatum bargaining experiments-A personal review. Journal of Economic Behavior and Organization 27, 329-344 (1995).

36. M. Elson, FlexibleMeasures. com: Competitive reaction time task. DOI: https://doi. org/10.17605/OSF. IO/4G7FV, (2016).

37. D. S. Chester, E. N. Lasko, Validating a standardized approach to the Taylor Aggression Paradigm. Social Psychological and Personality Science 10, 620-631 (2019).

38. G. Gan et al., Reward vs. retaliation-The role of the mesocorticolimbic salience network in human reactive aggression. Frontiers in behavioral neuroscience 10, 179 (2016). 
bioRxiv preprint doi: https://doi.org/10.1101/2020.09.29.318287; this version posted December 14, 2020. The copyright holder for this preprint (which was not certified by peer review) is the author/funder, who has granted bioRxiv a license to display the preprint in perpetuity. It is made available under aCC-BY-NC-ND 4.0 International license.

Mishor et al

39. S. da Cunha-Bang et al., Violent offenders respond to provocations with high amygdala and striatal reactivity. Social cognitive and affective neuroscience $12,802-$ 810 (2017).

40. A. P. Skibsted et al., Aggression-related brain function assessed with the Point Subtraction Aggression Paradigm in fMRI. Aggressive behavior 43, 601-610 (2017).

41. A. H. Buss, M. Perry, The aggression questionnaire. Journal of personality and social psychology 63, 452 (1992).

42. B. A. Golomb, M. Cortez-Perez, B. A. Jaworski, S. Mednick, J. Dimsdale, Point subtraction aggression paradigm: Validity of a brief schedule of use. Violence and victims 22, 95-103 (2007).

43. T. Allison, A. Puce, G. McCarthy, Social perception from visual cues: role of the STS region. Trends in cognitive sciences 4, 267-278 (2000).

44. J. Zaki, K. Hennigan, J. Weber, K. N. Ochsner, Social cognitive conflict resolution: contributions of domain-general and domain-specific neural systems. Journal of Neuroscience 30, 8481-8488 (2010).

45. K. A. Miczek et al., Neurobiology of escalated aggression and violence. Journal of Neuroscience 27, 11803-11806 (2007).

46. A. Raine, M. Buchsbaum, L. LaCasse, Brain abnormalities in murderers indicated by positron emission tomography. Biological psychiatry 42, 495-508 (1997).

47. K. J. Friston et al., Psychophysiological and modulatory interactions in neuroimaging. Neuroimage 6, 218-229 (1997).

48. M. Potegal, Temporal and frontal lobe initiation and regulation of the top-down escalation of anger and aggression. Behavioural brain research 231, 386-395 (2012).

49. J. Archer, Sex differences in aggression in real-world settings: A meta-analytic review. Review of general Psychology 8, 291-322 (2004).

50. C. F. Yang et al., Sexually dimorphic neurons in the ventromedial hypothalamus govern mating in both sexes and aggression in males. Cell 153, 896-909 (2013).

51. X. Xu et al., Modular genetic control of sexually dimorphic behaviors. Cell 148, 596607 (2012).

52. E. B. Keverne, Pheromones, vomeronasal function, and gender-specific behavior. Cell 108, 735-738 (2002).

53. M. L. Seghier, The angular gyrus: multiple functions and multiple subdivisions. The Neuroscientist 19, 43-61 (2013).

54. I. R. Olson, A. Plotzker, Y. Ezzyat, The Enigmatic temporal pole: a review of findings on social and emotional processing. Brain 130, 1718-1731 (2007).

55. E. Franzen, R. Myers, Neural control of social behavior: prefrontal and anterior temporal cortex. Neuropsychologia 11, 141-157 (1973).

56. I. Savic, H. Berglund, B. Gulyas, P. Roland, Smelling of odorous sex hormone-like compounds causes sex-differentiated hypothalamic activations in humans. Neuron 31, 661-668 (2001).

57. D. Joel et al., Sex beyond the genitalia: The human brain mosaic. Proceedings of the National Academy of Sciences 112, 15468-15473 (2015).

58. M. Okamoto, M. Shirasu, R. Fujita, Y. Hirasawa, K. Touhara, Child odors and parenting: a survey examination of the role of odor in child-rearing. PloS one 11, e0154392 (2016).

59. J. N. Lundström et al., Maternal status regulates cortical responses to the body odor of newborns. Frontiers in psychology 4, 597 (2013). 
60. T. Uebi et al., Sampling, identification and sensory evaluation of odors of a newborn baby's head and amniotic fluid. Scientific reports 9, 1-11 (2019).

61. T. I. Case, B. M. Repacholi, R. J. Stevenson, My baby doesn't smell as bad as yours: The plasticity of disgust. Evolution and Human Behavior 27, 357-365 (2006).

62. D. Maestripieri, Functional aspects of maternal aggression in mammals. Canadian Journal of Zoology 70, 1069-1077 (1992).

63. B. B. Svare, in Parental care in mammals. (Springer, 1981), pp. 179-210.

64. L. A. Ebensperger, Strategies and counterstrategies to infanticide in mammals. Biological Reviews 73, 321-346 (1998).

65. S. B. Hrdy, Infanticide among animals: a review, classification, and examination of the implications for the reproductive strategies of females. Ethology and Sociobiology 1, 13-40 (1979).

66. C. Dulac, L. A. O'Connell, Z. Wu, Neural control of maternal and paternal behaviors. Science 345, 765-770 (2014).

67. Y. Isogai et al., Multisensory logic of infant-directed aggression by males. Cell 175, 1827-1841. e1817 (2018).

68. K. Stern, M. K. McClintock, Regulation of ovulation by human pheromones. Nature 392, 177-179 (1998).

69. J. H. de Groot, M. A. Smeets, Human fear chemosignaling: evidence from a metaanalysis. Chemical senses 42, 663-673 (2017).

70. M. A. Smeets et al., Chemical fingerprints of emotional body odor. Metabolites 10, 84 (2020).

71. L. Stowers, T. F. Marton, What is a pheromone? Mammalian pheromones reconsidered. Neuron 46, 699-702 (2005).

72. T. D. Wyatt, The search for human pheromones: the lost decades and the necessity of returning to first principles. Proceedings of the Royal Society B: Biological Sciences 282, 20142994 (2015).

73. C. J. Wysocki, G. Preti, Facts, fallacies, fears, and frustrations with human pheromones. The Anatomical Record Part A: Discoveries in Molecular, Cellular, and Evolutionary Biology: An Official Publication of the American Association of Anatomists 281, 1201-1211 (2004).

74. M. Meredith, Human vomeronasal organ function: a critical review of best and worst cases. Chem Senses 26, 433-445 (2001).

75. G. B. Choi, D. J. Anderson, A nose by any other name (should smell as sweetly). Cell 123, 550-553 (2005).

76. U. Boehm, Z. Zou, L. B. Buck, Feedback loops link odor and pheromone signaling with reproduction. Cell 123, 683-695 (2005).

77. H. Yoon, L. Enquist, C. Dulac, Olfactory inputs to hypothalamic neurons controlling reproduction and fertility. Cell 123, 669-682 (2005).

78. M. J. Baum, J. A. Cherry, Processing by the main olfactory system of chemosignals that facilitate mammalian reproduction. Horm Behav 68, 53-64 (2015).

79. N. Kang, M. J. Baum, J. A. Cherry, A direct main olfactory bulb projection to the 'vomeronasal' amygdala in female mice selectively responds to volatile pheromones from males. Eur J Neurosci 29, 624-634 (2009).

80. F. Faul, E. Erdfelder, A.-G. Lang, A. Buchner, G* Power 3: A flexible statistical power analysis program for the social, behavioral, and biomedical sciences. Behavior research methods 39, 175-191 (2007). 
81. N. Sobel et al., Unexplained Repeated Pregnancy Loss is Associated with Altered Perceptual and Brain Responses to Men's Body-Odor. BioRxiv, (2020).

82. B. J. Bushman, R. F. Baumeister, A. D. Stack, Catharsis, aggression, and persuasive influence: Self-fulfilling or self-defeating prophecies? Journal of personality and social psychology 76, 367 (1999).

83. W. Güth, On ultimatum bargaining experiments-A personal review. Journal of Economic Behavior \& Organization 27, 329-344 (1995).

84. P. A. McGrath et al., A new analogue scale for assessing children's pain: an initial validation study. Pain 64, 435-443 (1996).

85. R. W. Levenson, P. Ekman, W. V. Friesen, Voluntary facial action generates emotionspecific autonomic nervous system activity. Psychophysiology 27, 363-384 (1990).

86. S. Baron-Cohen, S. Wheelwright, R. Skinner, J. Martin, E. Clubley, The autismspectrum quotient (AQ): Evidence from asperger syndrome/high-functioning autism, malesand females, scientists and mathematicians. Journal of autism and developmental disorders 31, 5-17 (2001).

87. C. Spielberger, R. Gorsuch, R. Lushene, P. Vagg, G. Jacobs, Manual for the state-trait anxiety scale. Consulting Psychologists, (1983).

88. T. Weiss et al., Human Olfaction without Apparent Olfactory Bulbs. Neuron 105, 3545. e35 (2020).

89. D. N. Greve, B. Fischl, Accurate and robust brain image alignment using boundarybased registration. Neuroimage 48, 63-72 (2009).

90. M. Jenkinson, S. Smith, A global optimisation method for robust affine registration of brain images. Medical image analysis 5, 143-156 (2001).

91. M. Jenkinson, P. Bannister, M. Brady, S. Smith, Improved optimization for the robust and accurate linear registration and motion correction of brain images. Neuroimage 17, 825-841 (2002).

92. J. Andersson, M. Jenkinson, S. Smith. (Oxford: University of Oxford, 2007).

93. J. L. Andersson, M. Jenkinson, S. Smith, Non-linear registration aka Spatial normalisation FMRIB Technial Report TR07JA2. FMRIB Analysis Group of the University of Oxford, (2007).

94. S. M. Smith, Fast robust automated brain extraction. Human brain mapping 17, 143155 (2002).

95. M. W. Woolrich, B. D. Ripley, M. Brady, S. M. Smith, Temporal autocorrelation in univariate linear modeling of FMRI data. Neuroimage 14, 1370-1386 (2001).

96. C. F. Beckmann, M. Jenkinson, S. M. Smith, General multilevel linear modeling for group analysis in FMRI. Neuroimage 20, 1052-1063 (2003).

97. M. W. Woolrich, T. E. Behrens, C. F. Beckmann, M. Jenkinson, S. M. Smith, Multilevel linear modelling for FMRI group analysis using Bayesian inference. Neuroimage 21, 1732-1747 (2004).

98. M. Woolrich, Robust group analysis using outlier inference. Neuroimage 41, 286-301 (2008).

99. K. J. Worsley, 14 Statistical analysis of activation images. Functional MRI: An introduction to methods, 251 (2001).

100. D. R. Gitelman, W. D. Penny, J. Ashburner, K. J. Friston, Modeling regional and psychophysiologic interactions in $\mathrm{fMRI}$ : the importance of hemodynamic deconvolution. Neuroimage 19, 200-207 (2003). 
bioRxiv preprint doi: https://doi org/10.1101/2020.09.29.318287; this version posted December 14,2020. The copyright holder for this preprint (which was not certified by peer review) is the author/funder, who has granted bioRxiv a license to display the preprint in perpetuity. It is made available under aCC-BY-NC-ND 4.0 International license.

Mishor et al

101. S. M. Smith et al., Advances in functional and structural MR image analysis and implementation as FSL. Neuroimage 23, S208-S219 (2004).

102. W. D. Penny, K. J. Friston, J. T. Ashburner, S. J. Kiebel, T. E. Nichols, Statistical parametric mapping: the analysis of functional brain images. (Elsevier, 2011).

103. R. C. Team, R: A language and environment for statistical computing. (2013). 